Algebraic $8 \mathcal{G}$ Geometric $\mathcal{T}$ opology

Volume 4 (2004) 961-1011

Published: 31 October 2004

ATG

\title{
A class of tight contact structures on $\Sigma_{2} \times I$
}

\author{
TANyA COFER
}

\begin{abstract}
We employ cut and paste contact topological techniques to classify some tight contact structures on the closed, oriented genus- 2 surface times the interval. A boundary condition is specified so that the Euler class of the of the contact structure vanishes when evaluated on each boundary component. We prove that there exists a unique, non-product tight contact structure in this case.
\end{abstract}

AMS Classification 57M50; 53C15

Keywords Tight, contact structure, genus-2 surface

\section{Introduction}

If $M$ is a compact, oriented 3 -manifold with boundary, a (positive) contact structure on $M$ is a completely non-integrable 2-plane distribution $\xi$ given as the kernel of a non-degenerate 1-form $\alpha$ such that $\alpha \wedge d \alpha>0$ at every point of $M$. We say $\xi$ is tight if there is no embedded disk $D \subset M^{3}$ with the property that $\xi$ is everywhere tangent to $D$ along $\partial D$. Such a $D$ is called an overtwisted disk and contact structures containing such disks are called overtwisted contact structures.

The field of contact topology has changed profoundly and developed rapidly during the last decades of the twentieth century. In the 1970's, Lutz and Martinet 22] showed that every closed, orientable three-manifold admits a contact structure. By the 1980's and early 1990's, results of Bennequin [1] and Eliashberg [6] were indicating the existence of a qualitative difference between the classes of tight and overtwisted contact structures. It was Eliashberg who made it clear that the topologically interesting case to study is tight contact structures [5]. He did this by showing that, up to isotopy, overtwisted contact structures are in one-to-one correspondence with homotopy classes of 2-plane fields on $M$. Soon after, he gave us the classifications of tight contact structures on $B^{3}$ (a foundational result for the classification of contact structures on three manifolds), $S^{3}, S^{2} \times S^{1}$ and $\mathbb{R}^{3}[\underline{6}$. A rush of further classification studies ensued, 
including the classification of tight contact structures on the 3-torus 19, lens spaces 17, 10, 12, solid tori, $T^{2} \times I$ [12, 21], torus bundles over circles [10, 13], and circle bundles over closed surfaces [11, 13. Etnyre and Honda [8] made a significant contribution by exhibiting a manifold that carries no tight contact structure whatsoever.

This critical mass of understanding has recently yielded a coarse classification principle for tight contact structures on 3-manifolds. Collectively, work by Colin, Giroux, Honda, Kazez and Matić 3, 4, 16] indicates that if $M$ is closed, oriented and irreducible, then $M$ supports finitely many isotopy classes of tight contact structures if and only if $M$ is atoroidal. In order to gain further understanding of the tight contact structures on atoroidal manifolds with infinite fundamental group, Honda Kazez and Matić studied hyperbolic 3-manifolds that fiber over the circle [17. In their case $M=\Sigma_{g} \times I$, where $\Sigma_{g}$ is the genus-g surface, $I=[0,1]$ and $\xi$ is "extremal" with respect to the Bennequin inequality. That is, $\left|e(\xi)\left[\Sigma_{g} \times\{t\}\right]\right|=2 g-2, t \in[0,1]$.

In the present paper, $\Sigma_{2}$ is a closed, oriented genus-2 surface and $M=\Sigma_{2} \times I$. We classify tight contact structures on $\left(M, \Gamma_{\partial M}, \mathcal{F}\right)$ where $\Gamma_{\partial M}$ is specified to be a single, nontrivial separating curve on each boundary component and $\mathcal{F}$ is a foliation which is adapted to this dividing set. This is a basic case of a boundary condition satisfying $e(\xi)\left[\Sigma_{2} \times\{0\}\right]=e(\xi)\left[\Sigma_{2} \times\{1\}\right]=0$.

Our classification exploits cut-and-paste methods developed by Honda, Kazez and Matić [16, 17] for constructing tight contact structures on Haken manifolds. The first step is to perform a Haken decomposition of $M$ in the contact category (called a convex decomposition). By keeping track of certain curves (dividing curves) on $\partial M$ and all cutting surfaces, we specify the contact structure in the complement of a union of 3-balls. Under certain conditions, we may then extend this contact structure to the interior of each ball so that the resulting structure is tight on the cut-open manifold. Since this type of decomposition can generally be done in a number of ways, we apply gluing theorems to determine which of these decompositions are associated to distinct tight contact structures on $M$.

Our first splitting of $M$ is along a convex annulus $A$ that separates $M$ into the disjoint union of two genus-2 handlebodies. Although there are an infinite number of possible dividing sets on $A$, a series of reduction arguments allow us to consider only four. We then apply the gluing/classification theorem [14] to a convex decomposition of each handlebody with each of the four dividing sets on $A$ in turn. These convex decompositions also allow us to locate bypasses along $A$, establishing equivalence among some of the contact structures supported on $M \backslash A$. This process yields an upper bound of two for the number of tight contact structures on $M$. 
At this point, it is necessary to decide whether or not the two tight contact structures on the split-open manifold $M \backslash A$ are associated with distinct tight contact structures on $M$. Since we must use a state-transition argument and the gluing/classification theorem in the first stage of the classification, we are unable to establish universal tightness for one of the two structures. This presents an obstacle to applying the gluing theorem directly. Thus, stage two of the classification is to adapt the gluing theorem [2, 16] and conclude the existence of a unique, non-product tight contact structure on $M$. This adaptation involves exploiting what we know about which bypasses exist along $A$ and constructing an infinite class of covers of $M \backslash A$ which we prove to be tight.

This process culminates in Lemma 3.0.4 and Theorem 4.2.4 which constitute the main focus of this paper. They are summarized in the following statement:

Theorem 1.0.1 (Main Theorem) There exists exactly one non-product tight contact structure on $\left(M, \Gamma_{\partial M}, \mathcal{F}\right)$ where $\Gamma_{\partial M}$ is specified to be a single, nontrivial separating curve on each boundary component and $\mathcal{F}$ is a foliation which is adapted to this dividing set.

Once this theorem is established, we then demonstrate a special property of the unique, non-product tight contact structure $\xi$ on $M$. If $(M, \xi)$ is contained in some $\left(M^{\prime}, \xi^{\prime}\right)$, then for any convex surface with boundary $S \subset M^{\prime}$ such that $\partial S \subset \partial M$ and $\#\left(\partial S \cap \Gamma_{\partial M}\right)=2$, the dividing set on $S$ cannot contain any boundary-parallel dividing arcs. This is because complementary bypasses always exist inside of $(M, \xi)$.

In the following sections, we describe the background results, tools and methods necessary for our classification.

\section{Background and tools}

\subsection{Convex surfaces}

We say that a curve $\gamma$ inside a contact manifold $(M, \xi)$ is Legendrian if it is everywhere tangent to $\xi$. Consider a properly embedded surface $S \subset(M, \xi)$. Generically, the intersection $\xi_{p} \cap T_{p} S$ at a point $p \in S$ is a vector $X(p)$. Integrating the vector field $X$ on $S$ gives us the a singular foliation called the characteristic foliation $\xi_{S}$. The leaves of the characteristic foliation are

Legendrian by definition. A surface $S \subset M$ is called convex if there exists a vector field $v$ transverse to $S$ whose flow preserves the contact structure $\xi$. Such 
a vector field is called a contact vector field. Given a convex surface $\mathrm{S}$, we define the dividing set $\Gamma_{S}=\{x \in S \mid v(x) \in \xi(x)\}$. Generically, this is a collection of pairwise disjoint, smooth closed curves (dividing curves) on a closed surface $S$ or a collection of curves and arcs if $S$ has boundary (where the dividing arcs begin and end on $\partial S)[9$. The curves and arcs of the dividing set are transverse to the characteristic foliation and, up to isotopy, this collection is independent of the choice of contact vector field $v$. Moreover, the union of dividing curves divides $S$ into positive and negative regions $R_{ \pm} \cdot R_{+}\left(R_{-}\right) \subset \partial M$ is the set of points where the orientation of $\xi$ agrees (disagrees) with the orientation of $S$.

Giroux proved that a properly embedded closed surface in a contact manifold can be $C^{\infty}$-perturbed into a convex surface [9]. We will refer to this as the perturbation lemma. If we want to keep track of the contact structure in a neighborhood of a convex surface, we could take note of the characteristic foliation. However, the characteristic foliation is very sensitive to small perturbations of the surface. Giroux Flexibility 9] highlights the usefulness of the dividing set $\Gamma_{S}$ by showing us that $\Gamma_{S}$ captures all of the important contact topological information in a neighborhood of $S$. Therefore, we can keep track of the dividing set instead of the exact characteristic foliation.

Given a singular foliation $\mathcal{F}$ on a convex surface $S$, a disjoint union of properly embedded curves $\Gamma$ is said to divide $\mathcal{F}$ if there exists some $I$-invariant contact structure $\xi$ on $S \times I$ such that $\mathcal{F}=\left.\xi\right|_{S \times\{0\}}$ and $\Gamma$ is the dividing set for $S \times\{0\}$.

Theorem 2.1.1 (Giroux Flexibility) Let $S \subset\left(M^{3}, \xi\right)$ be a convex surface in a contact manifold which is closed or compact with Legendrian boundary. Suppose $S$ has characteristic foliation $\left.\xi\right|_{S}$, contact vector field $v$ and dividing set $\Gamma_{S}$. If $\mathcal{F}$ is another singular foliation on $S$ which is divided by $\Gamma$, then there is an isotopy $\phi_{s}, s$ in $[0,1]$ of $S$ such that

(1) $\phi_{0}(S)=S$,

(2) $\left.\xi\right|_{\phi_{1}(S)}=\mathcal{F}$,

(3) $\phi$ fixes $\Gamma$,

(4) $\phi_{s}(S)$ is transverse to $v$ for all $s$.

The Legendrian realization principle specifies the conditions under which a collection of curves on a convex surface $S$ may be realized as a collection of Legendrian curves by perturbing $S$ to change the characteristic foliation on $S$ while keeping $\Gamma_{S}$ fixed. In general this is not a limiting condition. The result is 
achieved by isotoping the convex surface $S$ through surfaces that are convex with respect to the contact vector field $v$ for $S$ so that the collection of curves on the isotoped surface are made Legendrian.

Let $C$ be a collection of closed curves and arcs on a convex surface $S$ with Legendrian boundary. We call $C$ nonisolating if:

(1) $C$ is transverse to $\Gamma_{S}$.

(2) Every arc of $C$ begins and ends on $\Gamma_{S}$.

(3) The elements of $C$ are pairwise disjoint.

(4) If we cut $S$ along $C$, each component intersects the dividing set $\Gamma_{S}$.

An isotopy $\phi_{s}, s \in[0,1]$ of a convex surface $S$ with contact vector field $v$ is called admissible if $\phi_{s}(S)$ is transverse to $v$ for all $s$.

Theorem 2.1.2 (Legendrian Realization) If $C$ is a nonisolating collection of disjoint, properly embedded closed curves and arcs on a convex surface $S$ with Legendrian boundary, there is an admissible isotopy $\phi_{s}, s \in[0,1]$ so that:

(1) $\phi_{0}=i d$,

(2) each surface $\phi_{s}(S)$ is convex,

(3) $\phi_{1}\left(\Gamma_{S}\right)=\Gamma_{\phi_{1}(S)}$,

(4) $\phi_{1}(C)$ is Legendrian.

A useful corollary to Legendrian realization was formulated by Kanda [20]:

Corollary 2.1 Suppose a closed curve $C$ on a convex surface $S$

(1) is transverse to $\Gamma_{S}$,

(2) nontrivially intersects $\Gamma_{S}$.

Then $C$ can be realized as a Legendrian curve.

Suppose $\gamma \subset S$ is Legendrian and $S \subset M$ is a properly embedded convex surface. We define the Thurston-Bennequin number $t b\left(\gamma, F r_{S}\right)$ of $\gamma$ relative to the framing, $F r_{S}$, of $S$ to be the number of full twists $\xi$ makes relative to $S$ as we traverse $\gamma$, where left twists are defined to be negative. It turns out that $t b\left(\gamma, F r_{S}\right)=-\frac{1}{2} \#\left(\Gamma_{S} \cap \gamma\right)$ (see figure 1). When $\gamma$ is not a closed curve, we will refer to the twisting $t\left(\gamma, F r_{S}\right)$ of the arc $\gamma$ relative to $F r_{S}$.

Given any Legendrian curve $\gamma=\partial S$ with non-positive Thurston-Bennequin number, the following relative version of Giroux's perturbation lemma, proved 


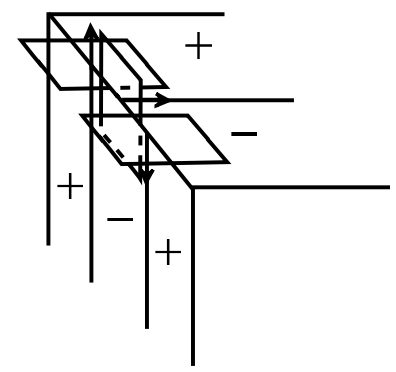

Figure 1: The dividing set on a cutting surface

by Honda [12, asserts that we can always arrange for the contact planes to twist monotonically in a left-handed manner as we traverse the curve. That is, an annular neighborhood $A$ of a Legendrian curve $\gamma$ with $t b(\gamma)=-n$ inside a contact manifold $(M, \xi)$ is locally isomorphic to $\left\{x^{2}+y^{2} \leq \epsilon\right\} \subset\left(\mathbb{R}^{2} \times\right.$ $\mathbb{R} / \mathbb{Z},(x, y, z))$ with contact 1 -form $\alpha=\sin (2 \pi n z) d x+\cos (2 \pi n z) d y, n \in \mathbb{Z}^{+}$ 12, 14. This is called standard form. Once this is achieved, it is then possible to perturb the surface $S$ so as to make it convex.

Theorem 2.1.3 (Relative Perturbation) Let $S \subset M$ be a compact, oriented, properly embedded surface with Legendrian boundary such that $t\left(\gamma, F r_{S}\right) \leq 0$ for all components $\gamma$ of $\partial S$. There exists a $C^{0}$ - small perturbation near the boundary which fixes $\partial S$ and puts an annular neighborhood $A$ of $\partial S$ into standard form. Then, there is a further $C^{\infty}$-small perturbation (of the perturbed surface, fixing $A$ ) which makes $S$ convex. Moreover, if $v$ is a contact vector field on a neighborhood of $A$ and transverse to $A$, then $v$ can be extended to a vector field transverse to all of $S$.

\subsection{The method: decomposing $(M, \xi)$}

We will be analyzing $\left(M^{3}, \xi\right)$ by cutting it along surfaces into smaller pieces and then analyzing the pieces. A familiar way to do this is to cut down $M$ along a sequence of incompressible surfaces $\left\{S_{i}\right\}$ into a union of balls. This is known as a Haken decomposition:

$$
M=M_{0} \stackrel{S_{1}}{\rightsquigarrow} M_{1} \stackrel{S_{2}}{\rightsquigarrow} \ldots \stackrel{S_{n}}{\rightsquigarrow} M_{n}=\amalg B^{3}
$$

In order to do define and exploit an analogous procedure in the contact category, we will need to make use of a couple of important results, including the following theorem, due to Eliashberg, which is a foundational result for the cut-and-paste methods described here: 
Theorem 2.2.1 (Eliashberg's Uniqueness Theorem) If $\xi$ is a contact structure in a neighborhood of $\partial B^{3}$ that makes $\partial B^{3}$ convex and the dividing set on $\partial B^{3}$ consists of a single closed curve, then there is a unique extension of $\xi$ to a tight contact structure on $B^{3}$ (up to isotopy that fixes the boundary).

Also, Giroux proved that we can determine when convex surfaces have tight neighborhoods by looking at their dividing sets 9 .

Theorem 2.2.2 (Giroux's Criterion) Suppose $S \neq S^{2}$ is a convex surface in a contact manifold $(M, \xi)$. There exists a tight neighborhood for $S$ if and only if $\Gamma_{S}$ contains no homotopically trivial closed curves. If $S=S^{2}$, then $S$ has a tight neighborhood if and only if $\# \Gamma_{S^{2}}=1$.

By Giroux's criterion, we know that if a homotopically trivial dividing curve appears on a convex surface inside a contact manifold, then our contact structure is overtwisted. If our surface happens to be $S^{2}$, then more than one dividing curve indicates an overtwisted contact structure.

Let $M$ be an irreducible contact 3-manifold with boundary. Then $M$ admits a Haken decomposition along incompressible surfaces $\left\{S_{i}\right\}$ [18. To do this in the contact category, we may take one of two approaches. In one approach, we assume $M$ carries a contact structure $\xi$ which makes $\partial M$ convex. In this case we may, at each stage of the decomposition, (1) use the relative perturbation lemma to perturb $S_{i}$ into a convex surface with Legendrian boundary, and (2) cut along $S_{i}$ and round edges (see the following discussion) so that the cut-open manifold is a smooth contact manifold with convex boundary [15].

Alternatively, we can do this decomposition abstractly along surfaces with divides with the goal of constructing a tight contact structure on $M$ (which may or may not exist). In this case, we begin with a convex structure $\left(M, \Gamma, R_{+}(\Gamma)\right.$, $\left.R_{-}(\Gamma)\right)$ [15] where $\Gamma, R_{+}(\Gamma)$ and $R_{-}(\Gamma)$ satisfy all the properties of a dividing set and positive and negative regions on $\partial M$ were $\partial M$ to be convex with respect to some contact structure $\xi$ on $M$. Then we can cut open $M$ along surfaces with divides $S_{i}$ that we hope will be convex with respect to the contact structure that we are attempting to construct. Note that we will make no distinction between these "pre-convex" surfaces and actual ones since the contact structure in a (contact) product neighborhood of the surface is determined up to isotopy by its dividing set. If we are able to decompose $M$ in this manner and we end up with a union of $\left(B^{3}, S^{1}\right)$ 's, then we may use Giroux flexibility and Eliashberg's uniqueness theorem to conclude the existence of a contact structure $\xi$ on $M$ which is compatible with the decomposition, and which is 
tight on the cut-open manifold. If there is a tight contact structure on $M$ which makes $\partial M$ convex, it is always possible to perform a convex decomposition of $(M, \Gamma)$ which is compatible with $\xi[15$. Hence, tight contact structures are determined by their convex decompositions.

Before we define this decomposition properly, we will explain what happens to the dividing sets on convex surfaces in a cut-open contact manifold when we smooth out the edges. We make use of the edge rounding lemma, a result that tells us what happens to the contact structure in a neighborhood of a Legendrian curve of intersection when we smooth away corners. A proof of the edge rounding lemma can be found in [12. A useful and brief discussion of the result can be found in 14. The following is a summary.

If we consider two compact, convex surfaces $S_{1}$ and $S_{2}$ with Legendrian boundary that intersect along a common Legendrian boundary curve, then a neighborhood of the common boundary is locally isomorphic to $\left\{x^{2}+y^{2} \leq \epsilon\right\} \subset$ $\left(\mathbb{R}^{2} \times \mathbb{R} / \mathbb{Z},(x, y, z)\right)$ with contact 1 -form $\alpha=\sin (2 \pi n z) d x+\cos (2 \pi n z) d y$, $n \in \mathbb{Z}^{+}$(standard form). We let $A_{i} \subset S_{i}, i \in\{1,2\}$ be an annular collar of the common boundary curve. It is possible to choose this local model so that $A_{1}=\{x=0,0 \leq y \leq \epsilon\}$ and $A_{2}=\{y=0,0 \leq x \leq \epsilon\}$. Then the two surfaces are joined along $x=y=0$ and rounding this common edge results in the joining of the dividing curve $z=\frac{k}{2 n}$ on $S_{1}$ to $z=\frac{k}{2 n}-\frac{1}{4 n}$ on $S_{2}$ for $k \in\{0, \ldots, 2 n-1\}$. See figure 2,

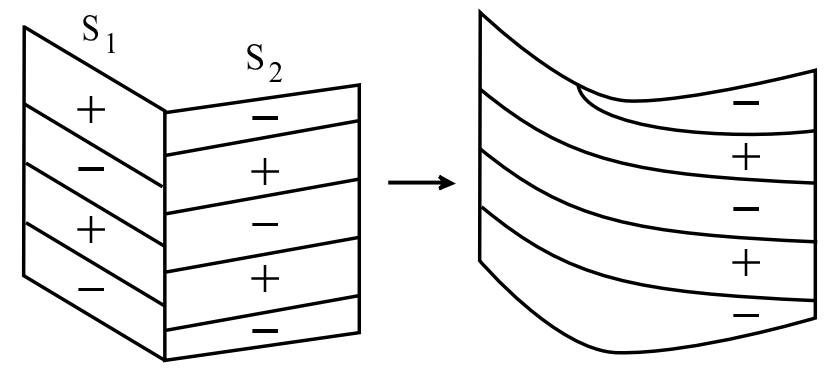

Figure 2: Edge Rounding

We are now ready to define our decomposition technique. We will use an abstract formulation for a convex decomposition in our classification. To do this, we will apply theorems such as Legendrian realization, relative perturbation, and edge rounding abstractly, taking care that our abstract divides satisfy the hypotheses of these theorems and the effect of applying of these theorems to the surfaces and divides follows as it would in the presence of an appropriate contact structure on $M$. 
Whenever it is possible to find such a decomposition into a union of 3-balls $B^{3}$ with $\# \Gamma_{\partial B^{3}}=1$, we say that

$$
(M, \Gamma)=\left(M_{0}, \Gamma_{0}\right) \stackrel{\left(S_{1}, \sigma_{1}\right)}{\rightsquigarrow}\left(M_{1}, \Gamma_{1}\right) \stackrel{\left(S_{1} 2, \sigma_{2}\right)}{\rightsquigarrow} \ldots \stackrel{\left(S_{n}, \sigma_{n}\right)}{\rightsquigarrow}\left(M_{n}, \Gamma_{n}\right)=\amalg\left(B^{3}, S^{1}\right)
$$

defines a convex decomposition of $(M, \Gamma)$ where the $\sigma_{i}$ are a set of divides on the convex surface $S_{i}$ and $M_{i}=M_{i-1} \backslash S_{i}$ inherits $\Gamma_{i}$ from $\Gamma_{i-1}, \sigma_{i}$ and (abstract) edge rounding.

Now, we would like to know how $\Gamma_{S_{i}}$ changes if we cut along a different, but isotopic, surface $S_{i}^{\prime}$ with the same boundary inside a contact manifold $(M, \xi)$. We may then return to our notion of a convex decomposition and apply the results abstractly to our cutting surfaces with divides. Honda and Giroux 10. 12. both studied the changes in the characteristic foliation on a convex surface under isotopy. Honda singled out the minimal, non-trivial isotopy of a cutting surface which he calls a bypass.

A bypass (figure 3) for a convex surface $S \subset M$ (closed or compact with Legendrian boundary) is an oriented, embedded half-disk $D$ with Legendrian boundary such that $\partial D=\gamma_{1} \cup \gamma_{2}$ where $\gamma_{1}$ and $\gamma_{2}$ are two arcs that intersect at their endpoints. $D$ intersects $S$ transversely along $\gamma_{1}$ and $D$ (with either its given orientation or the opposite one) has positive elliptic tangencies along $\partial \gamma_{1}$, a single negative elliptic tangency along the interior of $\gamma_{1}$, and only positive tangencies along $\gamma_{2}$, alternating between elliptic or hyperbolic. Moreover, $\gamma_{1}$ intersects $\Gamma_{S}$ in exactly the three elliptic points for $\gamma_{1}$ [12].

Isotoping a cutting surface past a bypass disk (figure 3) produces a change in the dividing set as shown in figure 4. Figure 4(A) shows how the dividing set changes if we attach a bypass above the surface. Figure 4(B) shows the change in the dividing set if we dig out a bypass below the surface. Formally, we have:

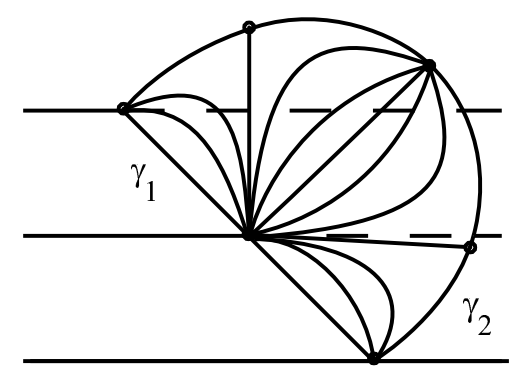

Figure 3: Bypass Disk

Theorem 2.2.3 (Bypass Attachment) Suppose $D$ is a bypass for a convex $S \subset M$. There is a neighborhood of $S \cup D$ in $M$ which is diffeomorphic 
to $S \times[0,1]$ with $S_{i}=S \times\{i\}, i \in\{0,1\}$ convex, $S \times[0, \epsilon]$ is $I$-invariant, $S=S \times\{\epsilon\}$ and $\Gamma_{S_{1}}$ is obtained from $\Gamma_{S_{0}}$ as in figure $4(A)$.

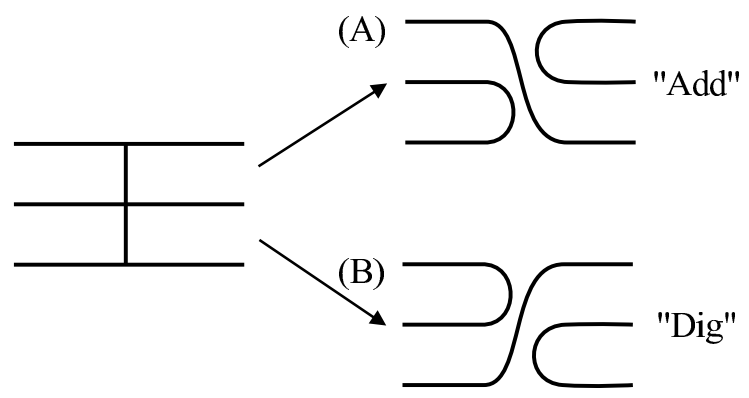

Figure 4: Abstract Bypass Moves

A useful result concerning bypass attachment is the bypass sliding lemma 13 , 17. It allows us some flexibility with the choice of the Legendrian arc of attachment.

Let $C$ be a curve on a convex surface $S$ and let $M=\min \left\{\#\left(C^{\prime} \cap \Gamma_{S}\right) \mid C^{\prime}\right.$ is isotopic to $C$ on $S\}$. We say that $C$ is efficient with respect to $\Gamma_{S}$ if $M \neq 0$ and the geometric intersection number $\#\left(C \cap \Gamma_{S}\right)=M$, or, if $M=0$, then $C$ is efficient with respect to $\Gamma_{S}$ if $\#\left(C \cap \Gamma_{S}\right)=2$.

Theorem 2.2.4 (Bypass Sliding Lemma) Let $R$ be an embedded rectangle with consecutive sides $a, b, c$ and $d$ on a convex surface $S$ so that $a$ is the arc of attachment of a bypass and $b, d \subset \Gamma_{S}$. If $c$ is a Legendrian arc that is efficient (rel endpoints) with respect to $\Gamma_{S}$, then there is a bypass for which $c$ is the arc of attachment.

\section{$2.3 \quad$ Gluing}

When we do a convex decomposition of $\left(M^{3}, \Gamma\right)$ and end up with a union of balls, each with a single dividing curve, we know we have a contact structure on $M$ which is tight on this cut-open manifold. If we glue our manifold back together along our cutting surfaces, the contact structure may not stay tight. It may be that an overtwisted disk $D \subset M$ intersected one or more of the cutting surfaces. There are two gluing theorems that tell us when we can expect the property of tightness to survive the regluing process.

One gluing theorem, which we will discuss in more detail later, is due to Colin [2], Honda, Kazez and Matić [16]. This theorem allows us to conclude tightness 
of the glued-up manifold when the dividing sets on the cutting surfaces are boundary-parallel. That is, the dividing set consists of arcs which cut off disjoint half-disks along the boundary. We will see that we must adapt this theorem in order to complete the classification in the case of our work here.

Theorem 2.3.1 (Gluing) Consider an irreducible contact manifold $(M, \xi)$ with nonempty convex boundary and $S \subset M$ a properly embedded, compact, convex surface with nonempty Legendrian boundary such that:

(1) $S$ is incompressible in $M$,

(2) $t\left(\delta, F r_{S}\right)<0$ for each connected component $\delta$ of $\partial S$ (i.e. each $\delta$ intersects $\Gamma_{\partial M}$ nontrivially), and

(3) $\Gamma_{S}$ is boundary-parallel.

If we have a decomposition of $(M, \xi)$ along $S$, and $\xi$ is universally tight on $M \backslash S$, then $\xi$ is universally tight on $M$.

The other gluing theorem is due to Honda [14. Honda discovered that, if we take a convex decomposition of an overtwisted contact structure on $M$ and look at all possible non-trivial isotopies (bypasses) of the cutting surfaces $S_{i}$, we will eventually come upon a decomposition that does not cut through the overtwisted disk.

In order to partition the set of contact structures on $M$ into isotopy classes of tight and overtwisted structures, we appeal to a mathematical algorithm known as the gluing/classification theorem. We describe this algorithm in the case where $M$ is a handlebody and $\xi$ is a contact structure on $M$ so that $\partial M$ is convex, since it is this case that is most relevant for our work here. One can find a statement of the general case in Honda [14.

Let $M=H_{g}$ be a handlebody of genus $g$. Prescribe $\Gamma_{\partial H_{g}}$ and a compatible characteristic foliation. Let $\left\{D_{i}\right\}, i \in\{1 \ldots g\}$ be a collection of disjoint compressing disks with $t b\left(\partial D_{i}, F r_{D_{i}}\right)<0$ yielding the convex splitting $M=H_{g} \rightsquigarrow H_{g} \backslash\left(D_{1} \cup D_{2} \cup \cdots \cup D_{g}\right)=B^{3}=M^{\prime}$. Also, let $C=\left(\Gamma_{1}, \Gamma_{2}, \cdots, \Gamma_{g}\right)$ represent a configuration of dividing sets on these disks, which we will call a state. We now need to decide if a given state corresponds to a tight contact structure on the original manifold $M$. We call a state $C$ potentially allowable (i.e. not obviously overtwisted) if $\left(M, \Gamma_{\partial M}\right)$, cut along $D_{1} \cup D_{2} \cup \cdots \cup D_{g}$ with configuration $C$, gives the boundary of a tight contact structure on $B^{3}$ (after edge rounding). That is, $\Gamma_{S^{2}}$ consists of a single closed curve.

Honda introduced the idea of the state transition and defined an equivalence relation on the set of configurations $\mathcal{C}_{M}$ on a manifold $M$ [14]. This equivalence 
relation partitions $\mathcal{C}_{M}$ into equivalence classes so that each equivalence class represents a distinct contact structure on $M$, either tight or overtwisted.

We say that a state transition $C \stackrel{s t}{\rightsquigarrow} C^{\prime}$ exists from a state $C$ to another state $C^{\prime}$ on $M$ provided:

(1) $C$ is potentially allowable.

(2) There is a nontrivial abstract bypass dig (see figure 4) from one copy of some $D_{i}$ in the cut-open $M^{\prime}$ and a corresponding add along the other copy of $D_{i}$ that produces $C^{\prime}$ from $C$ (a trivial bypass does not alter the dividing curve configuration).

(3) Performing only the dig from item (2) (above) does not change the number of dividing curves in $\Gamma_{\partial B^{3}}$ (i.e. the bypass disk exists inside of $M \backslash C$ ).

Define $\left\langle\sim>\right.$ on $\mathcal{C}_{M}$ as the equivalence relation generated by $\sim$, where $C \sim C^{\prime}$ if either $C \stackrel{s t}{\rightsquigarrow} C^{\prime}$ or $C^{\prime} \stackrel{s t}{\rightsquigarrow} C$. Then, an equivalence class under $<\sim>$ represents a tight contact structure provided every state in that class is potentially allowable (i.e. no state in the equivalence class is obviously overtwisted).

Theorem 2.3.2 (Gluing/Classification) The tight contact structures on $\left(H_{g}\right.$, $\left.\Gamma_{\partial H_{g}}\right)$ are in one-to-one correspondence with the equivalence classes under $<\sim>$ containing only potentially allowable states.

\subsection{State transition arguments}

The gluing/classification theorem for the case of $M=H_{g}$, as stated above, is reasonably practical. This is due to the fact that, although the number may be large, there are at least a finite number of possible states to check. In general, straightforward applications of gluing/classification theorem are impractical due to the possibility of an infinite number of dividing curve configurations (states) on general cutting surfaces. However, state transition arguments may still be used in a more general setting to show two contact structures are equivalent. Consider the case of our work here: $M=\Sigma_{2} \times I$ with fixed $\Gamma_{\partial M}$. Our classification begins by cutting $M$ along a convex annulus that separates $M$ into two genus-2 handlebodies. The infinite number of possible dividing curve configurations on the annulus precludes the use of the gluing/classification theorem on $M$. However, given two dividing curve configurations on the annulus, say $\Gamma_{A}$ $=A_{1}$ and $\Gamma_{A}=A_{2}$, we may conclude that (1) both configurations give the boundary of a tight contact structure on the cut-open manifold $M \backslash A$, and (2) contact structures on $\left(M \backslash A, A_{1}\right)$ and $\left(M \backslash A, A_{2}\right)$ come from the same contact 
structure on $M$, just cut along different, but isotopic annuli with the same boundary. This is achieved as follows:

(1) Consider contact structures on $\left(M \backslash A, A_{1}\right)$ and $\left(M \backslash A, A_{2}\right)$ specified by a convex decomposition of each, and suppose the gluing/classification theorem shows that the contact structure on $\left(M \backslash A, A_{1}\right)$ is tight.

(2) Consider the same cutting disks $D_{i}$ and $D_{i}^{\prime}, i \in\{1,2\}$ for both $\left(M \backslash A, A_{1}\right)$ and $\left(M \backslash A, A_{2}\right)$ with the contact structures specified above given by a choice of dividing set on each cutting disk. If there exists a bypass $B$ along $A_{1}$ (we must check this) so that isotoping past $B$ transforms $A_{1}$ into $A_{2}$ and simultaneously changes $\Gamma_{D_{i}}$ into $\Gamma_{D_{i}^{\prime}}$ for each $i$, then we say that there is a state transition taking $\left(A_{1}, \Gamma_{D_{1}}, \Gamma_{D_{2}}\right)$ to $\left(A_{2}, \Gamma_{D_{1}^{\prime}}, \Gamma_{D_{2}^{\prime}}\right)$. Any two contact structures related by a sequence of state transitions represent the same contact structure on $M$ just cut along different, but isotopic annuli with the same boundary. Note that this process establishes equivalence of contact structures on $M$, but not tightness. This issue will be dealt with separately.

\subsection{Some special bypasses}

Establishing the existence of bypasses requires work. In general, the bypasses along a convex surface $S$ inside a contact manifold $(M, \xi)$ may be "long" or "deep" (i.e. they exist outside of an $I$-invariant neighborhood), and establishing existence requires global information about the ambient manifold $M$. This is further evidenced by examining that the two Legendrian arcs, $\gamma_{1}$ and $\gamma_{2}$ (where $\gamma_{1} \subset S$ ) that comprise the boundary of the bypass half-disk $B$ (see figure 3). When we isotope the convex surface $S$ past $B$ to produce a new convex surface $S^{\prime}$, we see that $t\left(\gamma_{1}, F r_{S}\right)=-1$ whereas $t\left(\gamma_{2}, F r_{S^{\prime}}\right)=0$. Since a small neighborhood of a point on a Legendrian curve is isomorphic to a neighborhood

of the origin in $\mathbb{R}^{3}$ with the standard contact structure, the Thurston-Bennequin number can be decreased by one of the two moves in figure 5 , yet Benneqin's inequality tells us that $t b\left(\gamma, F r_{S}\right)$ is bounded above by the Seifert genus of $\gamma$. So, although it is easy to decrease twisting number, it not always possible to increase it.

There are two types of bypasses, however, that can be realized locally (in an I-invariant neighborhood of a convex surface): trivial bypasses and folding bypasses. Trivial bypasses are those that do not change the dividing curve configuration. Honda argues existence and triviality of trivial bypasses in [14, 


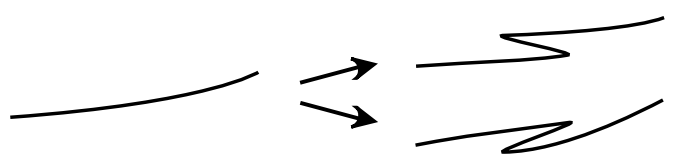

Figure 5: Decreasing $t b(\gamma)$

lemmas 1.8 and 1.9. Folding bypasses are the result of certain isotopies of a convex cutting surface inside an $I$-invariant neighborhood of the surface.

One way to establish existence of a bypass is to cut down the manifold $M$ into a union of 3-balls and invoke Eliahsberg's uniqueness theorem. Since there is a unique tight contact structure on the 3 -ball with $\# \Gamma_{\partial B^{3}}=1$ (rel boundary), only trivial bypasses exist in this case. Thus, if we can show that a proposed bypass along a convex surface $S \subset M$ is trivial on $B^{3}$, we can conclude it's existence.

A result related to trivial bypasses is the semi-local Thurston-Bennequin inequality. It is useful when we are attempting to distinguish between the product contact structure on $M$ and other structures. The following formulation is translated from Giroux [11]:

Proposition 2.1 (Semi-Local Thurston-Bennequin Inequality) Let $\xi$ be an $I$-invariant (product) contact structure on the product $U=S \times I$, where $I=[-1,1]$. Suppose $C$ is a simple closed curve on $S=S \times\{0\}$, and $\Gamma$ is a dividing set on $S$ which is adapted to $\xi_{S}$. Then, for all isotopies $\phi_{t}$ of $U$ which take $C$ to a Legendrian curve $\phi_{1}(C)$, the number of twists $\xi$ makes along $\phi_{1}(C)$ relative to the tangent planes to $\phi_{1}(S)$ satisfies the inequality

$$
t\left(\xi, F r_{\phi_{1}(S)}, \phi_{1}(C)\right) \leq-\frac{1}{2} \#(\Gamma, C)
$$

where $t\left(\xi, F r_{\phi_{1}(S)}, \phi_{1}(C)\right)$ measures the twisting of $\xi$ along $\phi_{1}(C)$ relative to $\mathrm{Fr}_{\phi_{1}(S)}$ and $\#(\Gamma, C)$ is $\min \left(\Gamma \cap C^{\prime}\right)$ where the minimum is taken over all closed curves $C^{\prime}$ isotopic to $C$ on $S$. Moreover, there is an isotopy which realizes equality.

Above, we mentioned that isotoping a convex surface $S$ past a bypass $B$ where $\partial B=\gamma_{1} \cup \gamma_{2}$ increases the twisting number $t\left(\gamma_{1}, F r_{S}\right)=-1$ to $t\left(\gamma_{2}, F r_{S^{\prime}}\right)=0$. So, if $C$ (containing $\gamma_{1}$ ) is a simple closed curve satisfying $\Gamma \cap C=\#(\Gamma, C$ ), then isotoping $S$ past $B$ causes the inequality to fail. Thus, it must be that the product structure can contain no non-trivial bypasses. 
We say that a closed curve is nonisolating if every component of $S \backslash \gamma$ intersects $\Gamma_{S}$. A Legendrian divide is a Legendrian curve such that all the points of $\gamma$ are tangencies. The ideas for the following exposition are borrowed from Honda et al. [16].

To produce a folding bypass, we must start with a nonisolationg closed curve $\gamma$ on a convex surface $S$ that does not intersect $\Gamma_{S}$. A strong form of Legendrian realization allows us to make $\gamma$ into a Legendrian divide. Then, there is a local model $N=S^{1} \times[-\epsilon, \epsilon] \times[-1,1]$ with coordinates $(\theta, y, z)$ and contact form $\alpha=d z-y d \theta$ in which the convex surface $S$ intersects the model as $S^{1} \times[-\epsilon, \epsilon] \times\{0\}$ and the Legendrian divide $\gamma$ is the $S^{1}$ direction (see figure 6).

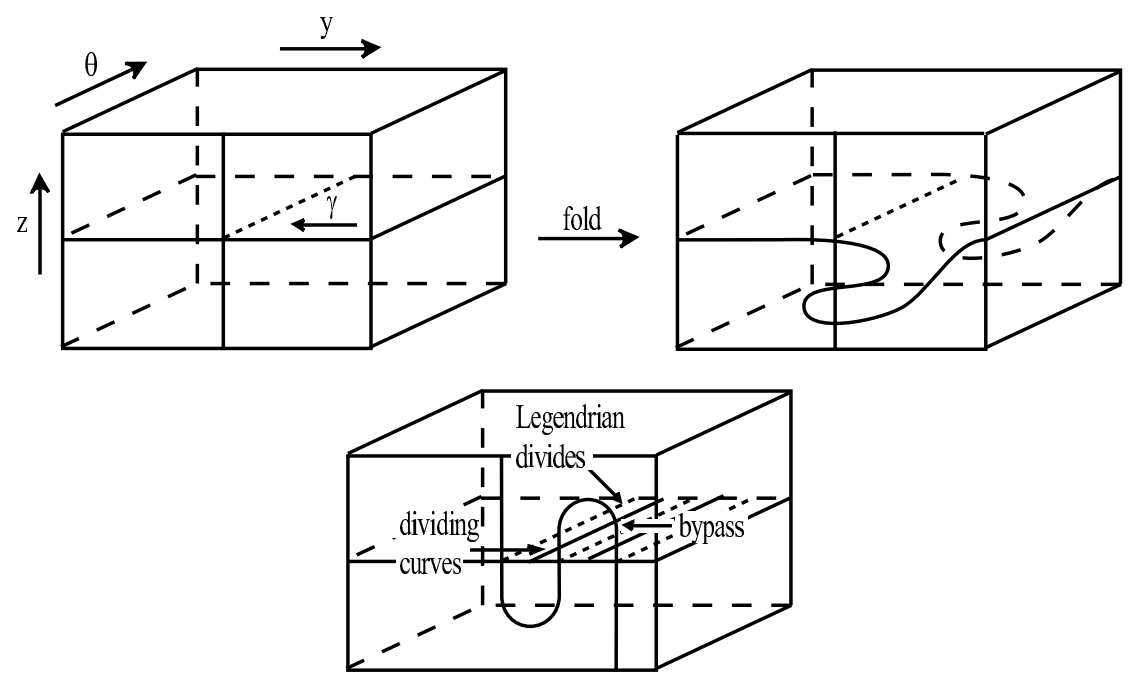

Figure 6: Folding

To fold around the Legendrian divide $\gamma$, we isotope the surface $S$ into an "S"shape (see the figure) inside of the model $N$. Outside of the model, $S$ and $S^{\prime}$ are identical. The result is a pair of dividing curves on $S^{\prime}$ parallel to $\gamma$. Note that the obvious bypass add indicated in figure [6 "undoes" the fold. It turns out that we can view the fold as an isotopy of the dividing set followed by a bypass dig as illustrated in figure 7 where the bypass dig is the one dual to the bypass add in figure 6 .

In many of the applications that follow, we will be establishing the existence of folding bypasses on solid tori. Note that, on the boundary of a solid torus with $2 n$ dividing curves, there are $2 n$ Legendrian divides spaced evenly between 


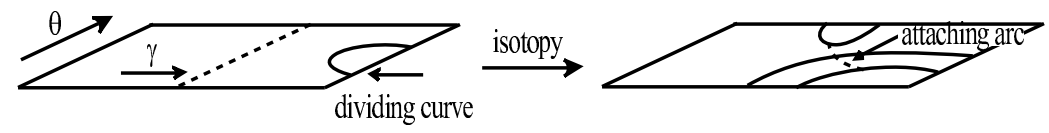

Figure 7: Folding is isotopy plus bypass dig

the dividing curves. Thus, there is always a Legendrian divide located near an existing dividing curve.

\section{Characterizations of potentially tight contact struc- tures on $\Sigma_{2} \times I$ with a specified configuration on the boundary}

We investigate the number of tight contact structures on $M=\Sigma_{2} \times I$ with a specific dividing set on the boundary $\partial\left(\Sigma_{2} \times I\right)=\Sigma_{0} \cup \Sigma_{1}$. The dividing set we specify on the boundary consists of a single separating curve $\gamma_{i}$ on each $\Sigma_{i}$, $i \in\{0,1\}$. These curves are chosen so that $\chi\left(\left(\Sigma_{i}\right)_{+}\right)=\chi\left(\left(\Sigma_{i}\right)_{-}\right)$. Here, $\left(\Sigma_{i}\right)_{ \pm}$ $i \in\{0,1\}$ represent the positive and negative regions of $\Sigma_{i} \backslash \Gamma_{\Sigma_{i}}$. The position of the $\gamma_{i}$ are indicated in figure 8

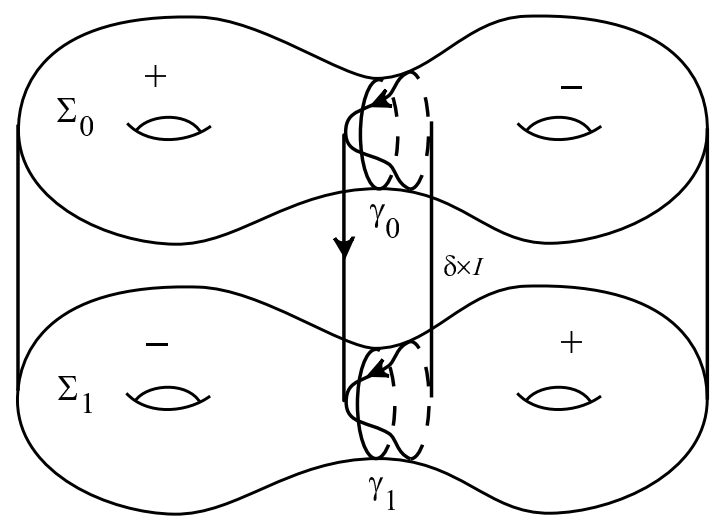

Figure 8: $\mathrm{M}=\Sigma_{2} \times I$

Our first strategy is to provide a convex decomposition of $M$ and partition the set of contact structures into equivalence classes using Honda's gluing/classification theorem [14. One difficulty here is that the first cutting surface $(\delta \times I$ as indicated in figure 8) of the decomposition is an annulus $A$. Fortunately, the 
infinitely many possibilities for $\Gamma_{A}$ fall into three natural categories (see figure 9). It is immediately clear in this case that $\Gamma_{A}$ of type $T 2_{k}^{-}$are overtwisted. For the following reduction arguments, we will assume that there is a tight contact structure on $M$ with $\Gamma_{A}$ as specified, and we prove that we can always find a bypass along $A$ so that isotoping $A$ past this bypass produces the desired reduction. We can assume that $\delta \times I$ is convex with Legendrian boundary since $\delta$ intersects $\Gamma_{\partial M}$ nontrivially. $M_{1}=M \backslash A=M_{1}^{+} \cup M_{1}^{-}$is given at the top of figure 10. We show first that all tight $T 2_{2 k+1}^{+}, T 1_{k}$, and $T 1_{-k}$ for $k \geq 1$ can be reduced to $T 2_{1}^{+}, T 1_{1}$, and $T 1_{-1}$, respectively.

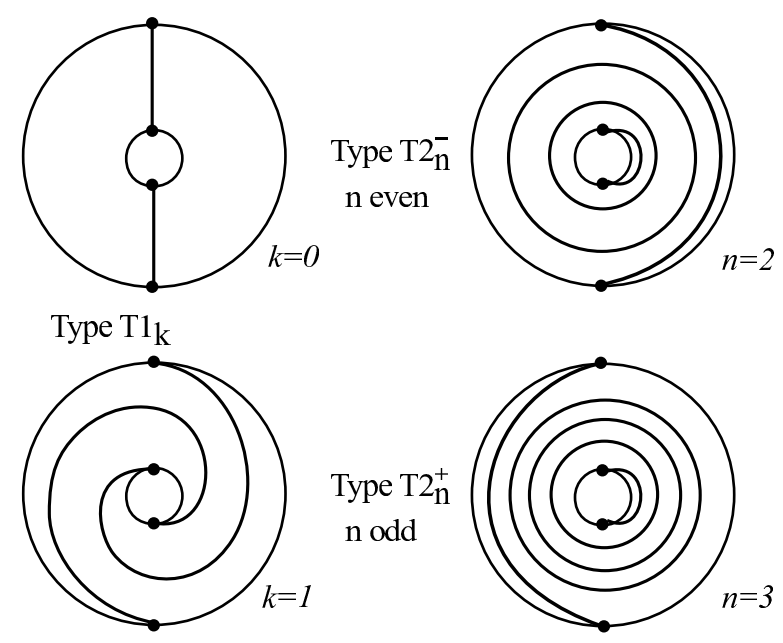

Figure 9: Classification of $\Gamma_{A}$

Lemma 3.0.1 $T 2_{2 k+1}^{+}, k \in \mathbb{Z}^{+}$can be reduced to $T 2_{1}^{+}$.

Proof Suppose there is a tight contact structure on $M$ with $\Gamma_{A}=T 2_{2 k+1}^{+}$, $k \geq 1$. Cut the $A_{+}$component of $M_{1}$ open along the convex cutting surface $\epsilon=\delta_{1} \times I$ where $\delta_{1}$ is positioned as in figure 10. We see that all but two possibilities for $\Gamma_{\epsilon_{+}}$contain a dividing curve straddling one of the positions 2 through $2 k+2$ or $2 k+5$ through $4 k+5$ as shown in figure [1]. Isotoping $A_{+}$across any of these bypasses yields a dividing set on the isotoped annulus equivalent to $T 2_{2 k-1}^{+}$.

The remaining possibilities are pictured. Both possibilities $i$ and $i i$ give us a dividing set on $M_{2} \cong S^{1} \times D^{2}=M_{1}^{+} \backslash \epsilon$ consisting of $2 k+4$ longitudinal curves as shown in figure [1]. If we choose a convex, meridional cutting surface $\eta$, we see, as in figure [12, that all possibilities for $\Gamma_{\eta_{+}}$contain a dividing curve 


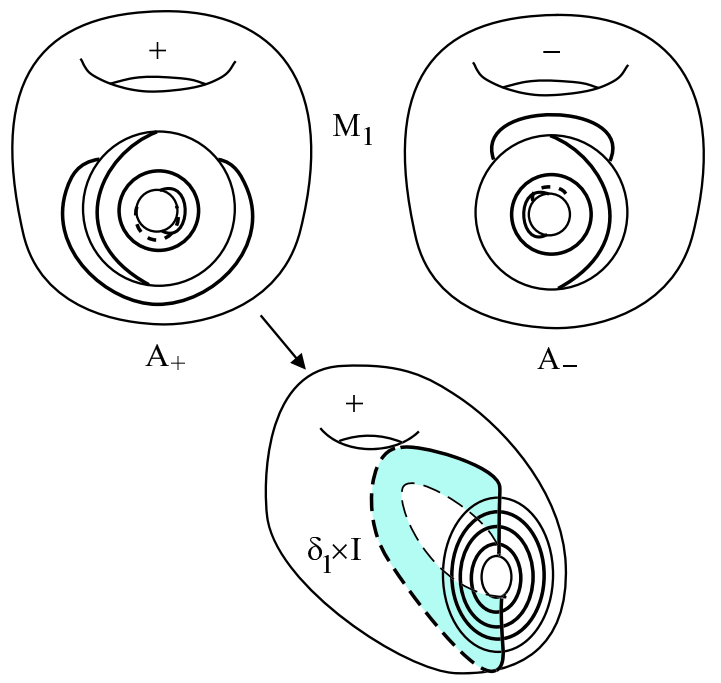

Figure 10: $M_{1}=M \backslash A$

straddling one of the positions 2 through $2 k+2$ for $k \geq 1$. Isotoping $A_{+}$across any of these bypasses yields a dividing set on the isotoped annulus equivalent to $T 2_{2 k-1}^{+}$. Thus, $T 2_{2 k+1}^{ \pm}, k \in \mathbb{Z}^{+}$can be reduced to $T 2_{1}^{ \pm}$.
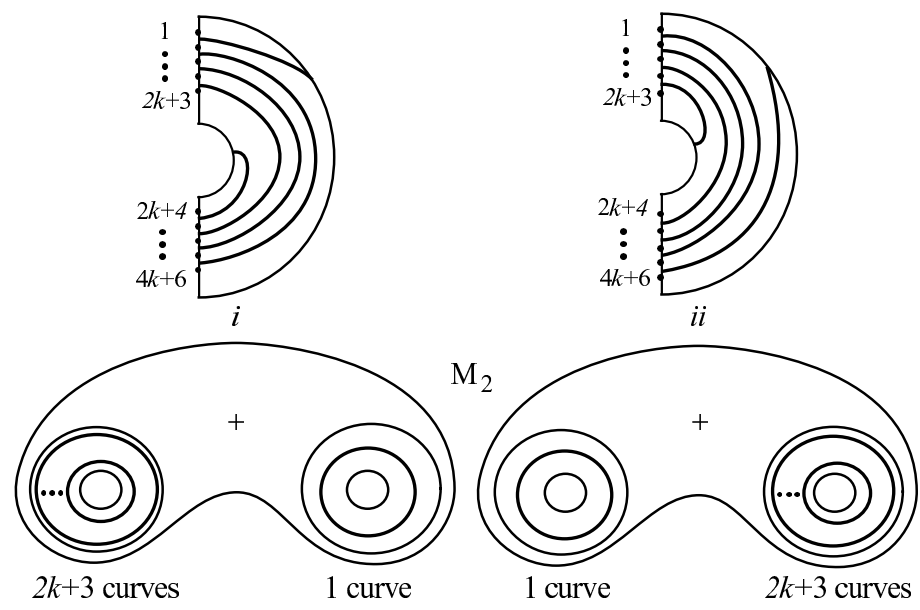

Figure 11: Convex decomposition for $M$ with $\Gamma_{A}=T 2_{2 k+1}^{+}$ 

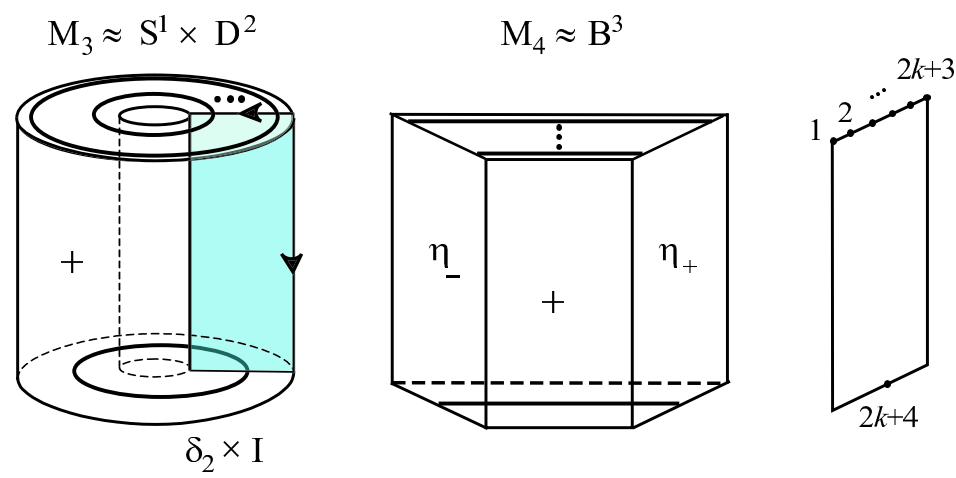

Figure 12: $\Gamma_{A}$ of type $T 2_{2 k+1}^{+}$

Lemma 3.0.2 $T 1_{k}, k \neq 0 \in \mathbb{Z}$ can be reduced to $T 1_{ \pm 1}$.

Proof Suppose there is a tight contact structure on $M$ with $\Gamma_{A}=T 1_{k}, k \leq$ -2 . We show that there is a bypass along $A$ so that isotoping $A$ past this bypasses produces a dividing set on the isotoped annulus equivalent to $T 1_{k+1}$. In this way $T 1_{k}, k \leq-2$ can be reduced to $T 1_{-1}$. The proof for positive $k$ is analogous.

Consider the proposed bypass indicated in figure 13. After rounding edges, we see that this bypass is trivial. Although pictured for $T 1_{-2}$, this is the case for all $k \leq-2$. Isotoping $A_{+}$across this bypass yields a dividing set on the isotoped annulus equivalent to $T 1_{k+1}$.

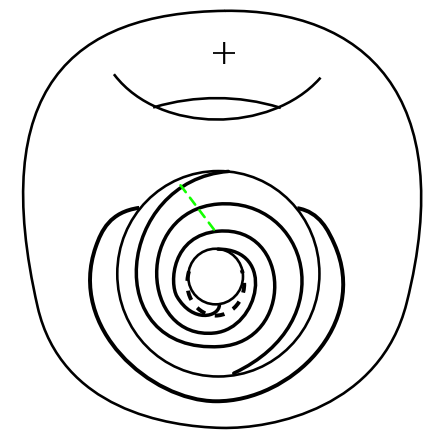

$\mathrm{A}_{+}$

Figure 13: Convex decomposition for $M$ with $\Gamma_{A}=T 1_{-k}$ 
Now we will turn our attention to constructing potentially tight contact structures on $M$ by further decomposing (abstractly) $M_{1}=M \backslash A$ with $\Gamma_{A}$ of type $T 1_{0}, T 1_{1}, T 1_{-1}$ and $T 2_{1}^{+}$. For each of these four possible $\Gamma_{A}$ in turn, we will apply the gluing theorem [2, 16] or partition the constructed contact structures into equivalence classes of tight and overtwisted structures on $M_{1}$ by applying the gluing/classification theorem for handlebodies. Further equivalences will be established on $M$ by locating state transitions along $A$. Gluing across the annulus will be addressed in the following section.

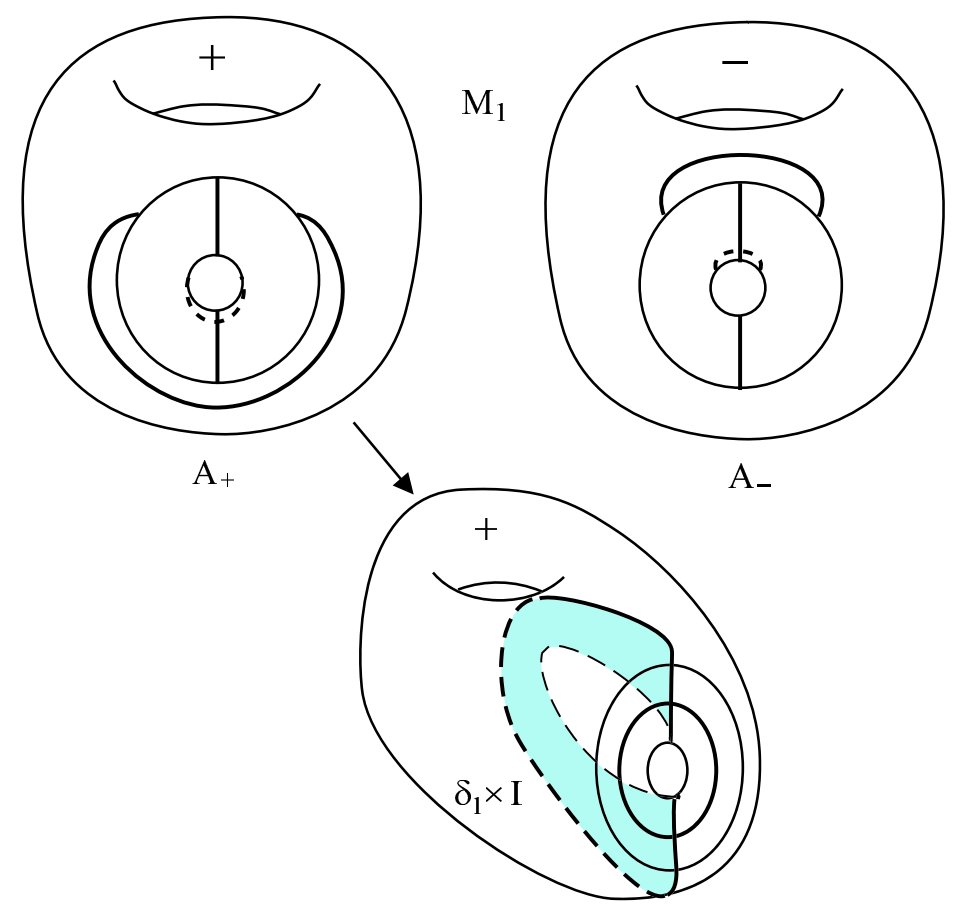

Figure 14: Convex decomposition of $M$ with $\Gamma_{A}=T 1_{0}(1)$

Lemma 3.0.3 There is a unique potentially tight contact structure on $M$ of type $T 1_{0}, T 1_{1}$ and $T 1_{-1}$. These contact structures are all equivalent on $M$ via state transitions. Moreover, they are universally tight on $M_{1}=M \backslash A$.

Proof Consider $M_{1}=M \backslash A$ with $\Gamma_{A}=T 1_{0}$ as pictured in figure 14] We show the decomposition for the component of $M_{1}$ containing $A_{+}\left(M_{1}^{+}\right)$. The argument for $M_{1}^{-}$is virtually identical. 


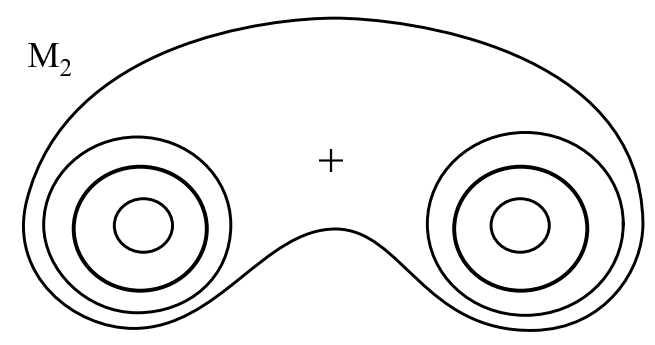

Figure 15: Convex decomposition of $M$ with $\Gamma_{A}=T 1_{0}(2)$

We cut open $M_{1}^{+}$along the convex cutting surface $\epsilon=\delta_{1} \times I$ with Legendrian boundary as indicated at the bottom of figure [14. Two copies of the cutting surface, $\epsilon_{+}$and $\epsilon_{-}$appear on the cut-open manifold $M_{2}=M_{1} \backslash \epsilon$. Since $t b\left(\partial \epsilon_{ \pm}\right)=-1$, there is only one tight possibility for $\Gamma_{\epsilon}$. Assuming this choice for $\Gamma_{\epsilon}$ and rounding edges along $\partial \epsilon_{ \pm}$, we get $\left(M_{2} \cong S^{1} \times D^{2}, \Gamma_{\partial_{M_{2}}}\right)$ as in figure 15.

We further decompose $M_{2}$ by cutting along a convex, meridional cutting surface $\eta$. Since $t b\left(\partial \eta_{ \pm}\right)=-1$, there is only one tight possibility for $\Gamma_{\eta}$. Assuming this choice for $\Gamma_{\eta}$ and rounding edges along $\partial \eta_{ \pm}$, we get $M_{3} \cong B^{3}$ with $\# \Gamma_{\partial_{M_{3}}}=1$. By Eliashberg's uniqueness theorem, there is a unique, universally tight contact structure on $M_{3} \cong B^{3}$ which extends the one on the boundary. Moreover, since the dividing sets on our cutting surfaces $\epsilon$ and $\eta$ are boundary-parallel, we may apply the gluing theorem [2, 16] (theorem 2.3.1) to conclude the existence of a unique, universally tight contact structure on $M_{1}$ with $\Gamma_{A}=T 1_{0}$. If we consider $M_{1}$ with $\Gamma_{A_{+}}=T 1_{ \pm 1}$ and round edges along $\partial A_{ \pm}$, we see that we

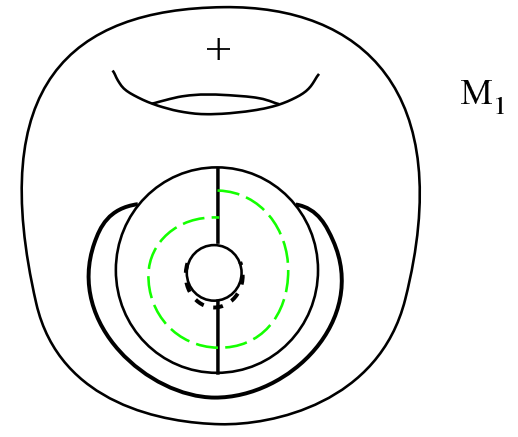

$\mathrm{A}_{+}$

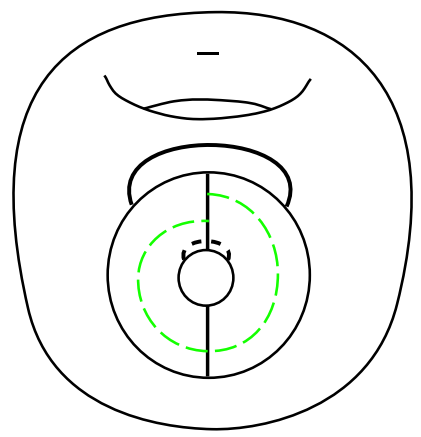

A-

Figure 16: $M_{1}=M \backslash A$ 


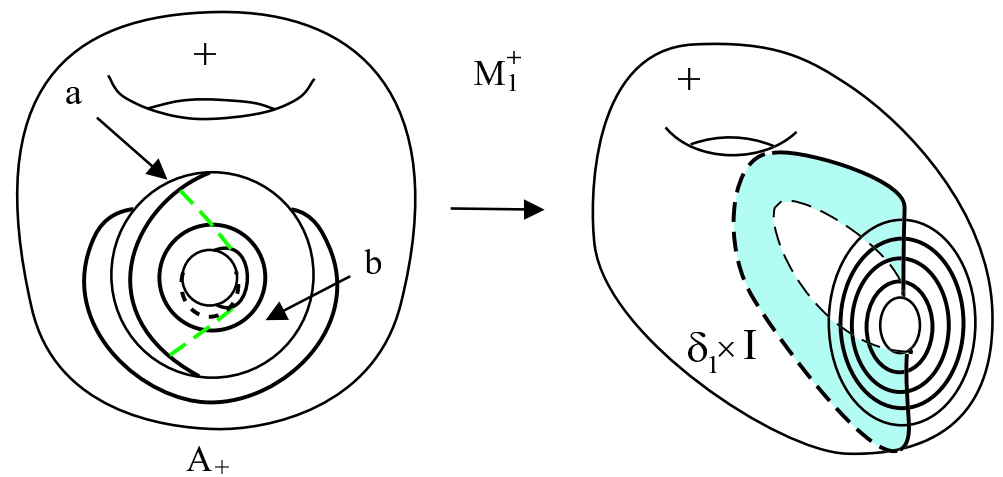

Figure 17: $M_{1}^{+}=M_{1} \backslash \epsilon$ with $\Gamma_{A_{+}}=T 2_{1}^{+}$and possible bypasses

obtain $M_{1}$ with a single homotopically essential closed dividing curve as in the $T 1_{0}$ case (see figure 14). Proceeding with the convex decomposition as in the $T 1_{0}$ case shows that there is a unique, universally tight contact structure on $M_{1}$ with $\Gamma_{A_{+}}=T 1_{1}$ and another with $\Gamma_{A_{+}}=T 1_{-1}$.

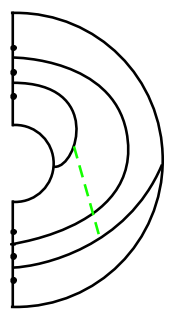

$i$

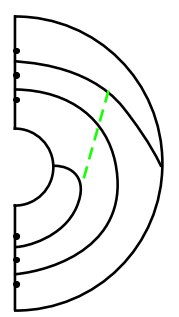

ii

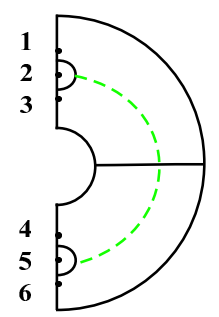

iii

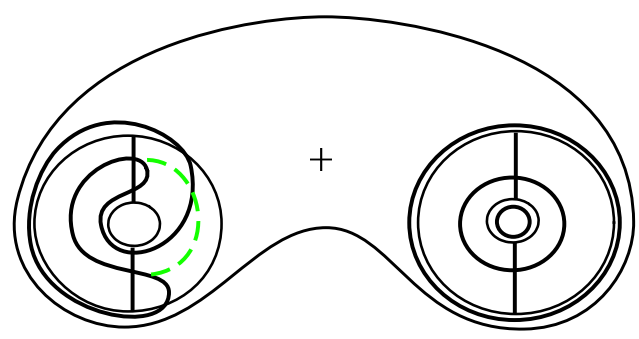

Figure 18: $M_{2}=M_{1} \backslash \epsilon$ with possible $\Gamma_{\epsilon}$ 
(A)

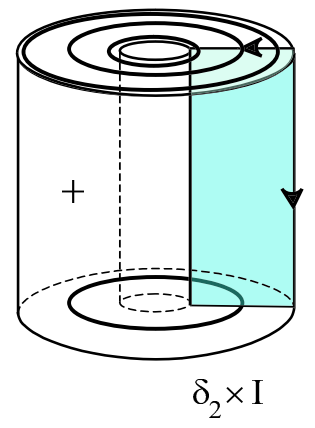

(B)
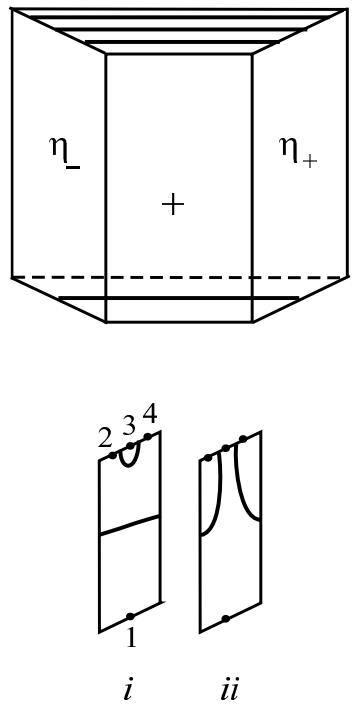

(C)

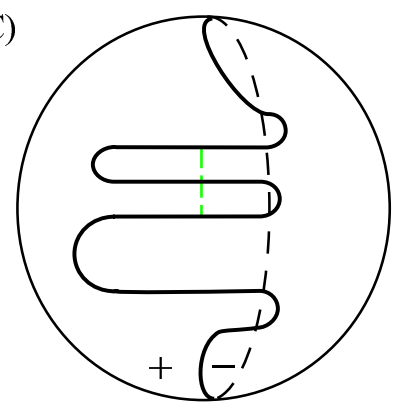

(D)

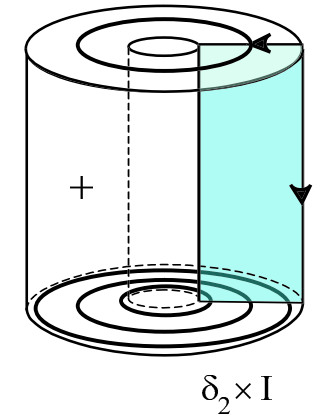

(E)
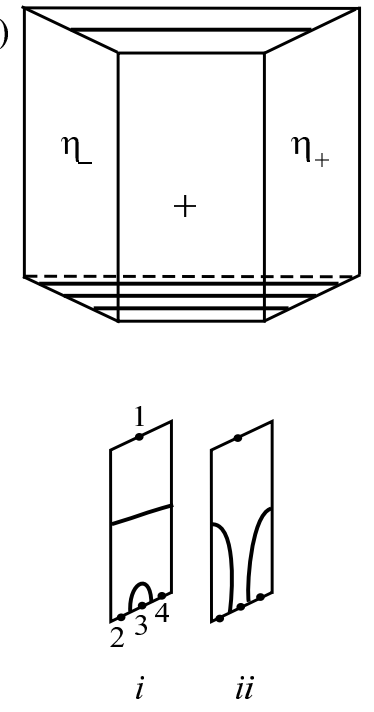

(F)

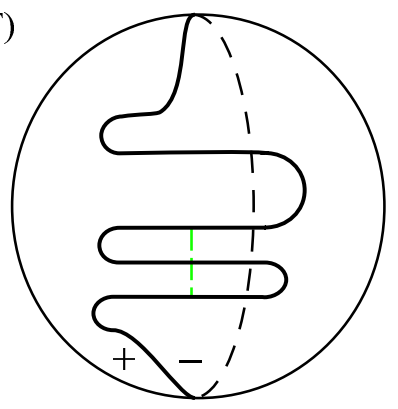

Figure 19: Unique Non-Product $M_{2}=M_{1} \backslash \epsilon$ with $\epsilon=i$ and $i i$ 
Now we show the equivalence of the tight contact structures on $M_{1}$ with $\Gamma_{A_{+}}=T 1_{0}, T 1_{1}$ and $T 1_{-1}$ by showing there exists a state transition along $A$ transforming the unique, universally tight contact structure on $M_{1}$ with $\Gamma_{A_{+}}=T 1_{0}$ into the unique universally tight contact structure on $M_{1}$ with $\Gamma_{A_{+}}=T 1_{1}$ and another transforming it into the unique universally tight contact structure on $M_{1}$ with $\Gamma_{A_{+}}=T 1_{-1}$. Let us consider $M_{1}$ with $\Gamma_{A_{+}}=T 1_{0}$ as in figure 16. The proposed bypasses along $A_{+}$and $A_{-}$are trivial, and, hence, exist. They transform $T 1_{0}$ into $T 1_{1}$ along $A_{+}$and into $T 1_{-1}$ along $A_{-}$while simultaneously transforming the dividing sets on $\epsilon$ and $\eta$ for the decomposition in the $T 1_{0}$ case into the dividing sets on $\epsilon$ and $\eta$ for the decomposition in the $T 1_{1}$ and $T 1_{-1}$ cases, respectively.

By lemma 5.2 of [17], there is a unique universally tight contact structure on $M \backslash A\left(\amalg_{i=1}^{2}\left(S_{i} \times I\right), S_{i} \cong T^{2} \backslash \nu(p t)\right)$ with $\Gamma_{A}=T 1_{0}$, and it is given by perturbing the foliation of $M \backslash A$ by leaves $S \times\{t\}, t \in[0,1]$. Recall that we have fixed a characteristic foliation adapted to the boundary-parallel dividing set $\Gamma_{\partial(M \backslash A)}$. The structure we describe here is the induced contact structure in a product neighborhood of our disjoint union of punctured tori such that $\mathcal{L}_{\frac{\partial}{\partial t}}(\xi)=0$. That is, flowing in the $I$-direction preserves $\xi$. This gives a dividing set on $A$ equivalent to $T 1_{0}$. We will call this ( $I$ - invariant) contact structure the product structure. Hence, lemma 3.0.3 states that the unique, potentially tight structures on $M$ with $\Gamma_{A}=T 1_{ \pm 1}$ are isotopic to the product structure.

Lemma 3.0.4 There is a unique, non-product potentially tight contact structure on $M$ of type $T 2_{1}^{+}$. This contact structure is tight on $M_{1}=M \backslash A$.

Proof We will make the argument for the $A_{+}$component of $M_{1}\left(M_{1}^{+}\right)$since the argument for the other component is completely analogous.

Suppose $\Gamma_{A}=T 2_{1}^{+}$. Note that if bypass $a$ indicated in figure 17 exists, there is a decomposition of $M$ along an annulus $A^{\prime}$, isotopic to $A$, with $\Gamma_{A^{\prime}}=T 1_{0}$. If bypass $b$ indicated in figure 17 exists, there is a decomposition of $M$ along an annulus $A^{\prime \prime}$, isotopic to $A$, with $\Gamma_{A^{\prime \prime}}=T 1_{-1}$. We first decompose $M_{1}$ in order to isolate a potentially tight contact structure that does not obviously contain one of these bypasses.

Let $\epsilon=\delta_{1} \times I$ be the convex cutting surface with Legendrian boundary indicated in figure 17. Cutting $M_{1}$ open along this cutting surface yields $M_{2}=M_{1} \backslash \epsilon$ with two copies $\epsilon_{+}$and $\epsilon_{-}$of the cutting surface. All but the three choices of $\Gamma_{\epsilon_{+}}$given in figure 18 immediately lead to a homotopically trivial dividing 
curve, and, hence, by Giroux's criterion, to the existence of an overtwisted disk. The boundary-parallel dividing curves of choice iii may be realized along $A_{+}$as bypasses $a$ and $b$ transforming $T 2_{1}^{+}$into $T 1_{0}$ and $T 1_{-1}$. We want to show that there is a unique potentially tight potentially non-product structure on $M$ with $\Gamma_{\epsilon_{+}}=i$ and another with $\Gamma_{\epsilon_{+}}=i i$, and that the state transition indicated in choice $i$ of figure 18 exists and takes one to the other. We will also show that there is no state transition with bypass attaching arc indicated in choice $i i$ of figure 18 taking the unique potentially tight potentially non-product structure with $\Gamma_{\epsilon_{+}}=i i$ into any structure with $\Gamma_{\epsilon_{+}}=i i i$. We then show there is, in fact, a single potentially tight contact structure on $M$ with $\Gamma_{A}=T 2_{1}^{+}$ and $\Gamma_{\epsilon_{+}}=i i i$ containing bypasses $a$ and $b$ as in figure 17. Finally we show that if the bypass indicated in figure 18 iii exists inside $M_{2}$, then isotoping $\epsilon$ past this bypass transitions this structure into an obviously overtwisted one with $\Gamma_{\epsilon}=i$. The state-transition argument for possible bypass digs along the corresponding dividing sets for $\Gamma_{\epsilon_{-}}$is similar. This exhausts all potential states and state transitions on $M_{1}$ with $\Gamma_{A}=T 2_{1}^{+}$in this equivalence class under gluing/classification (theorem 2.3.2 [14]).

Suppose we have $M_{2}$ with $\Gamma_{\epsilon_{+}}=i$. After rounding edges, we get $M_{2} \cong S^{1} \times D^{2}$ with four longitudinal dividing curves (see figure 18). Cutting $M_{2}$ open along a convex, meridional cutting surface $\eta=\delta_{2} \times I$ as in figure[19](A) yields $M_{3} \cong B^{3}$ with two copies of the cutting surface, $\eta_{+}$and $\eta_{-}$. Since $t b\left(\partial \eta_{+}\right)=-2$, there are two possibilities for $\Gamma_{\eta_{+}}$(see figure 19 (B)). One choice contains a boundaryparallel dividing arc straddling position 3 . This indicates the existence of a bypass half-disk $B$ that can be realized along $A_{+}$. Isotoping $A_{+}$across $B$ produces a dividing set on the isotoped annulus equivalent to $T 1_{0}$. Applying the remaining choice and rounding edges leads to $\# \Gamma_{\partial B^{3}}=1$ as in figure 19 (C). By Eliashberg's uniqueness theorem, there is a unique extension of this contact structure to the interior of $B^{3}$. Since the dividing set on $\eta$ is boundary-parallel, we may apply the gluing theorem (theorem 2.3.1 2, 16]), we can conclude that this contact structure is tight on $M_{2}$ with $\epsilon=i$. If we similarly decompose $M_{2}$ with $\Gamma_{\epsilon_{+}}=i i$ as in the figures $19(\mathrm{D})$ through $(\mathrm{F})$, we see that there is a unique, potentially tight contact structure (that is not obviously isotopic to a structure with a cut of type $\left.\Gamma_{A}=T 1_{0}\right)$ on $M$ that is tight on $M_{2}$ with $\Gamma_{\epsilon_{+}}=i i$.

Now, consider the proposed state transition from choice $i$ to choice $i i$ of $\Gamma_{\epsilon_{+}}$ on $M_{2}$. The bypass indicated on the left of figure 20 (A) is a folding bypass on $M_{2} \cong S^{1} \times D^{2}$, and such bypasses always exist [16]. We need to show that if we dig the bypass from $\epsilon_{+}$and glue it back along $\epsilon_{-}$, we transform the potentially tight potentially non-product $T 2_{1}^{+}$with $\Gamma_{\epsilon_{+}}=i$ into the unique potentially non-product $T 2_{1}^{+}$with $\Gamma_{\epsilon_{+}}=i i$. To see this, we need to use a slightly different 


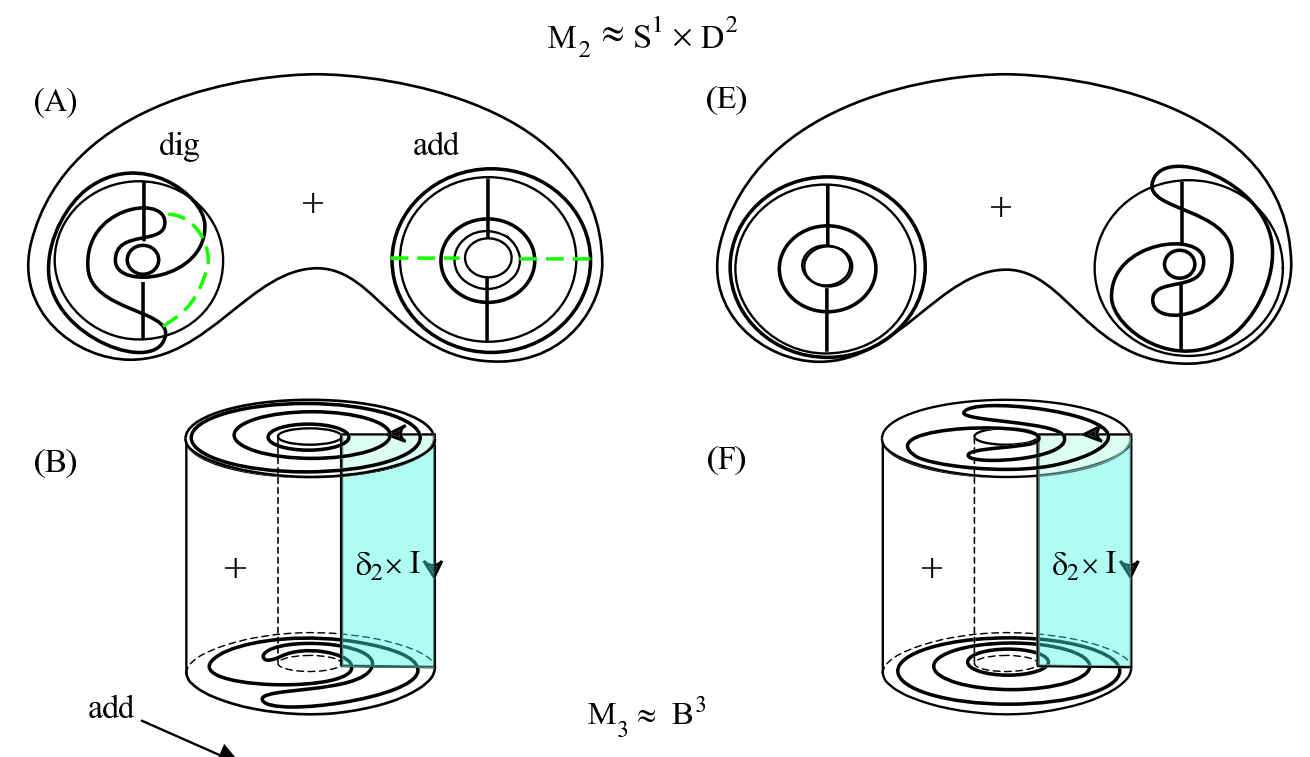

(C)

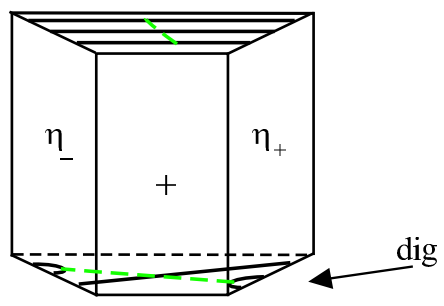

(D)

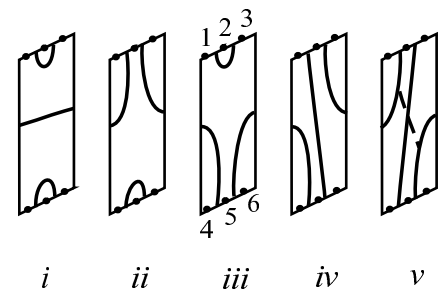

(G)

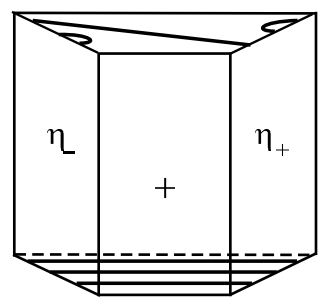

(H)

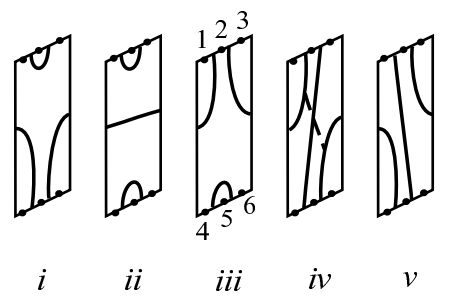

Figure 20: Equivalence of $M_{2}=M_{1} \backslash \epsilon$ with $\epsilon=i$ and $i i$

decomposition. Our new decomposition for $M_{2}$ with $\Gamma_{\epsilon_{+}}=i$ appears in figures 20 (A) through (D). Our new decomposition for $M_{2}$ with $\Gamma_{\epsilon_{+}}=i i$ appears in figures 20 (E) through $(\mathrm{H})$. In order to avoid cutting through the proposed bypass attaching arc, our first cutting surface (see figures 20 (B) and (F)) will be a convex cutting surface with Legendrian boundary that is not efficient. 
In this decomposition, we first would like to isolate the potentially tight potentially non-product contact structure from the previous decomposition. By cutting $M_{2}$ inefficiently along $\eta=\delta_{2} \times I$, we have five choices for $\Gamma_{\eta_{+}}$as in figures 20 (D) and (H). Choices $i$ and $i$ for both decompositions lead to a homotopically trivial dividing curve and hence, by Giroux's criterion, an overtwisted disk. In each decomposition, choice iii contains a boundary-parallel dividing curve (straddling positions 2 and 5, respectively). We conclude the existence of a bypass half-disk that can be realized on $A_{+}$in each decomposition, transforming $\Gamma_{A_{+}}$into $T 1_{0}$ or $T 1_{-1}$. The structures represented by choices $i v$ and $v$ are equivalent by the state transitions indicated in figures 20 (D) and (H). That is, (1) Both choices $i v$ and $v$ in figure (D) yield, after edge rounding, a single dividing curve on $\partial B^{3},(2)$ the abstract bypass dig indicated in figure (D) $v$ transforms $v$ into $i v$, and (3) performing the bypass dig of figure (D) $v$ does not change $\# \Gamma_{\partial B^{3}}$ (i.e. the bypass disk exists inside the cut-open manifold). The same is true for figures $(\mathrm{H}) i v$ and $v$. They represent the unique potentially non-product $T 2_{1}^{+}$with $\Gamma_{\epsilon_{+}}=i$ and the unique potentially non-product $T 2_{1}^{+}$ with $\Gamma_{\epsilon_{+}}=i i$, respectively (see figure 19).

Now that we have identified the potentially tight potentially non-product structure of figure 19 under this new decomposition, we wish to show the existence of the state transition along $\epsilon_{+}$with $\Gamma_{\epsilon_{+}}=i$. Note that digging the (folding) transitioning bypass from a portion of $\epsilon_{+}$on $M_{3} \cong B^{3}$ and gluing it back along a portion of $\epsilon_{-}$as in figure 20 (C) transforms the unique potentially tight potentially non-product structure on $M$ with $\Gamma_{\epsilon_{+}}=i$ and $\eta_{+}=i v$ into the unique potentially tight potentially non-product structure on $M$ with $\Gamma_{\epsilon_{+}}=i i$ and $\eta_{+}=v$. This establishes equivalence.

We know from the previous decomposition that the potentially tight potentially non-product contact structure on $M$ with $\Gamma_{\epsilon_{+}}=i$ or $i i$ is tight on $M_{2}$. We now show that the potential state transition taking $\Gamma_{\epsilon_{+}}=i i$ into any structure with $\Gamma_{\epsilon_{+}}=i i i$ does not exist (see figures 18 $i i$, 19 (F) and 21). From our choice of $\eta_{+}=i i$, we know that there is a bypass on the solid torus $M_{2}$ straddling position 4 as in figure 19 (E). This is equivalent to adding a bypass straddling position 3 along the outside of the torus (see the Attach=Dig property, p.64-66 of [16]). Since both this add and the proposed transitioning bypass cannot exist inside a tight manifold, we can conclude that the state transition from $\Gamma_{\epsilon_{+}}=i i$ to $\Gamma_{\epsilon_{+}}=i i i$ does not exist. Note that by this reasoning, we may conclude that bypasses $a$ and $b$ in figure 17 cannot exist in this case.

There is a single potentially tight structure on $M_{1}$ with $\Gamma_{A}=T 2_{1}^{+}$and $\Gamma_{\epsilon_{+}}=i i i$, and it is pictured in figure 22. All other potential $\Gamma_{\eta}$ are obviously overtwisted after edge-rounding. Clearly this choice of $\Gamma_{\epsilon_{+}}$indicates the 


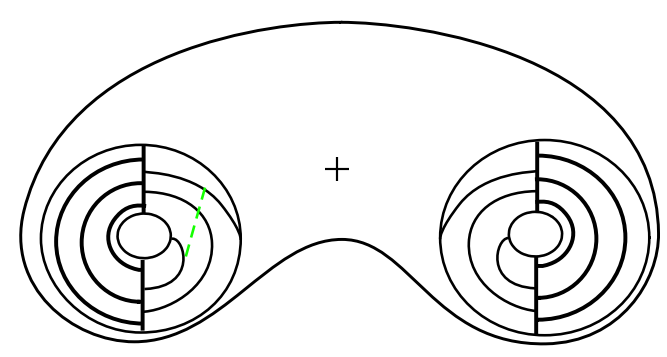

Figure 21: Non-Equivalence of $M_{2}=M_{1} \backslash \epsilon$ with $\epsilon=i i$ and $i i i$

existence of a bypass half-disk that can be realized along $A$. Isotoping $A$ past this bypass transforms $\Gamma_{A}=T 2_{1}^{+}$into $\Gamma_{A}=T 1_{0}$.

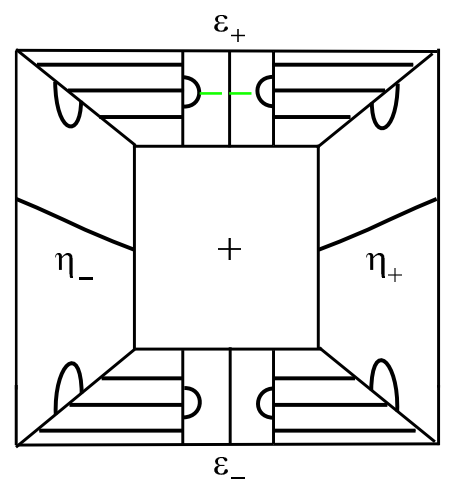

Figure 22: Non-Equivalence of $M_{2}=M_{1} \backslash \epsilon$ with $\epsilon=i i i$ and $i$

Suppose that the bypass dig from $\epsilon_{+}$, as indicated in figure 22, exists (see also figure $18 \mathrm{iii}$ ). From the figure, we see that digging the bypass from $\epsilon_{+}$and gluing back along $\epsilon_{-}$transforms this structure into an overtwisted one with $\Gamma_{\epsilon_{+}}=i$, not into the potentially tight structure of figures 19 (A) through (C) and 20 (A) through (D). The state-transition argument for possible bypass digs along the corresponding dividing sets for $\Gamma_{\epsilon_{-}}$is similar.

Having checked all possible states and state transitions on $M_{1}$ with $\Gamma_{A}=T 2_{1}^{+}$, we conclude, by Honda's gluing/classification theorem [14] (theorem 2.3.1), that the potentially tight potentially non-product structure on $M_{1}$ with $\Gamma_{A_{+}}=T 2_{1}^{+}$ is tight on $M_{1}$. This contact structure is potentially allowable on $M$.

By the semi-local Thurston-Bennequin inequality (see [11, and proposition 2.1] of this paper) we know that there can be no non-trivial bypasses inside a contact product neighborhood of a surface. Note that $M$ contains a $\partial$-parallel arc 
as in figure 23 indicating the existance of a bypass half-disk abutting $\partial M$. Isotoping $\Sigma_{0}=\Sigma_{2} \times\{0\}$ past this bypass half-disk results in a dividing set on the new, isotoped surface $\Sigma_{0}^{\prime}$ as in figure 24. Since this non-trivial bypass exists along $\partial M$, we may conclude that this structure is, in fact, not the product structure.

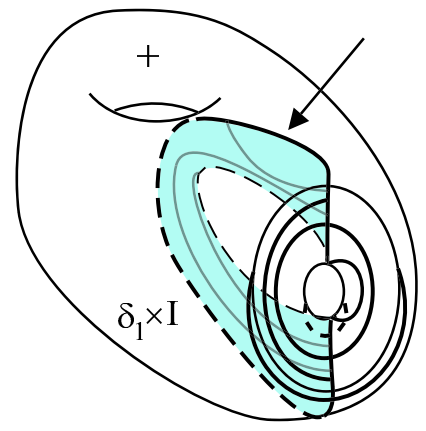

$\mathrm{A}_{+}$

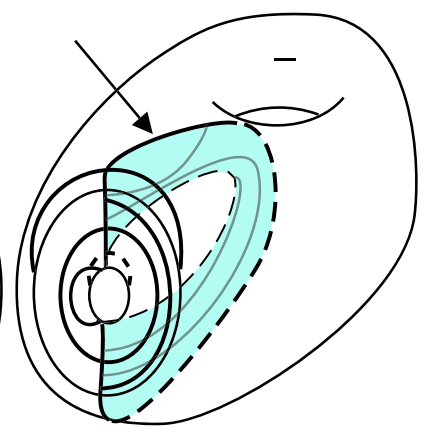

A-

Figure 23: $\mathrm{M}$ contains a non-trivial bypass along $\Sigma_{0}$

We now turn to the task of showing that this contact structure comes from a unique, non-product tight contact structure on $M$.

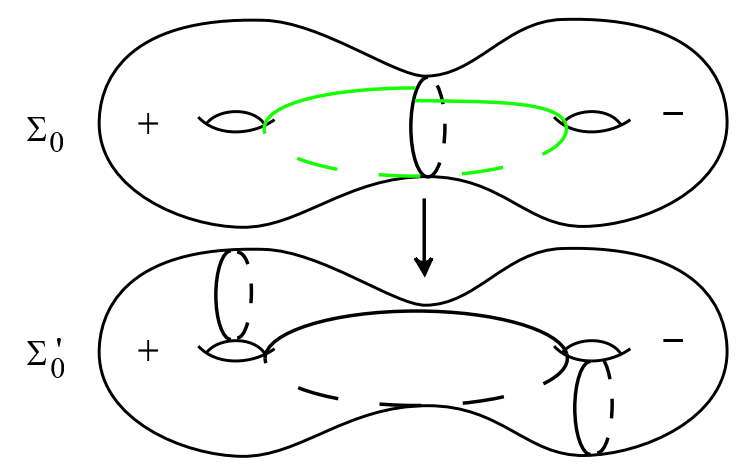

Figure 24: Isotoping $\Sigma_{0}$ past a non-trivial bypass

\section{Gluing}

It is now necessary to establish the tightness of the two potentially allowable (non-product and product) contact structures of the previous section. The 
strategy here will be to use the ideas involved in the proof of the gluing theorem for contact manifolds with convex boundary and boundary-parallel dividing curves on all gluing surfaces. This proof was originally given by Colin [2] and subsequently formulated in terms of convex decompositions by Honda et al. [16]. We say the dividing set $\Gamma_{S}$ on a convex surface is boundary-parallel if $\Gamma_{S}$ is a collection of arcs connecting $\partial S$ to $\partial S$ and this collection of arcs cuts off disjoint half-disks along the boundary of $S$. A contact structure $\xi$ on $M$ is universally tight if the pull-back $(\tilde{M}, \tilde{\xi})$ of the contact structure to any cover of $M$ is tight. The general statement was given earlier (the "gluing theorem" theorem 2.3.1).

There are two main obstacles to applying the gluing theorem directly. First of all, the dividing set $T 2_{1}^{+}$on $A$ is not boundary-parallel. So, we cannot use the theorem directly to $M_{1}$ glued along $A$. In the gluing theorem, the boundaryparallel requirement is necessary in order to guarantee that any bypass along the gluing surface at most introduces a pair of parallel dividing curves. However, we know from our decomposition precisely which bypasses exist along $A$. In the non-product case, they are trivial bypasses and folding bypasses along the central homotopically non-trivial closed dividing curve of $T 2_{1}^{+}$, which introduce a pair of dividing curves parallel to the original curve.

The second obstacle is that it is necessary in our case to use a state transition argument in establishing tightness of the non-product contact structure on $M_{1}=M \backslash A$. This argument relies on Honda's gluing/classification theorem which guarantees tightness but not universal tightness. Thus, we cannot pull our contact structure back to an arbitrary cover and expect that the structure remains tight. Instead, we will construct explicit covers $\tilde{M}_{i}$ of $M \backslash A$ and compute pull-back structures directly in order to establish tightness of $\left(\tilde{M}_{i}, \tilde{\xi}\right)$. Therefore, it is possible to establish the conditions necessary to apply the ideas of the gluing theorem and conclude tightness of $(M, \xi)$.

The idea of the proof here, following the proof of the gluing theorem, will be as follows. First, we will construct finite covers of $M_{1}$ in which all of the aforementioned folding bypasses are trivial and prove that these covers with the pull-back contact structures are tight. Then, we will assume the existence of an overtwisted disk $D$ inside $\mathrm{M}$ and look at controlled pull-backs of the bypasses necessary to push $A$ off of $D$ to the specified tight covers. In this manner, we will construct a cover $(\tilde{M}, \tilde{\xi})$, a pull-back of $\tilde{A}$ of $A$ and a lift $\tilde{D}$ of the overtwisted disk $D$. In this cover, all the bypasses needed to isotope $\tilde{A}$ off of $\tilde{D}$ will be trivial. Using tightness of $(\tilde{M}, \tilde{\xi})$, we can derive a contradiction to the existence of $D$, thereby establishing tightness of $M$. 


\subsection{Constructing tight covers}

Let us begin by constructing a $3: 1$ cover $\tilde{S}$ of the punctured torus as in figure 25 . Since this cover has a single boundary component, it must be a once punctured surface $\Sigma_{g}$ for some $g \in \mathbb{Z}^{+}$. An Euler characteristic calculation tells us that this cover $\tilde{S}$ must be $\Sigma_{2}-\nu(p t)$ :

$$
\begin{aligned}
\chi(\tilde{S}) & =1-2 g \\
& =3(1-2(1)) \\
& =-3
\end{aligned}
$$

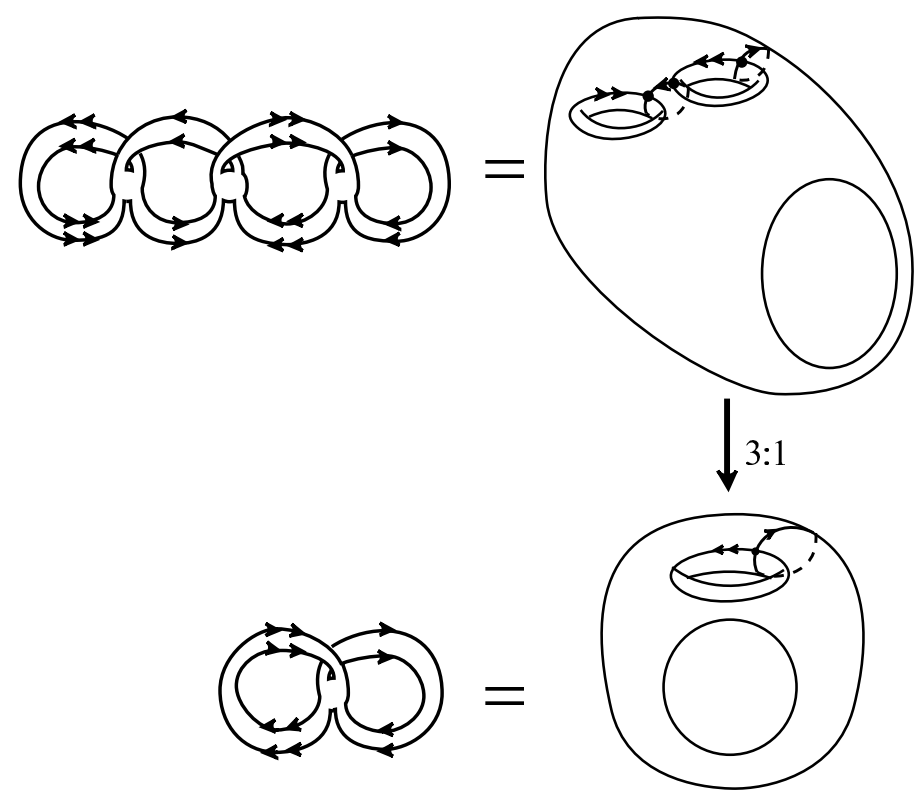

Figure 25: A covering space for $T^{2}-\nu(p t)$

Thickening each surface by crossing with an interval induces a cover of the punctured torus cross $I$ by $\left(\Sigma_{2}-\nu(p t)\right) \times I$. Thus, we have constructed a $3: 1$ cover of each component $M_{1}=M \backslash A$. Now, we use this construction and the fundamental domain in figure 26 to construct a 3:1 cover of $\left(\Sigma_{2}-\nu(p t)\right) \times I$. In this way, we construct a $3^{n}: 1$ cover of $M_{1}$ for each $n \in \mathbb{Z}^{+}$.

We now have a $m$ :1-fold covering space $\left(\tilde{M}=\Sigma_{m+1} \times I, p\right)$ of $M=\Sigma_{2} \times I$ such that $m=3^{n}$ for each $n \in \mathbb{Z}^{+}$. The restriction of such a cover to $M_{1}$ is the disjoint union of two copies of $\left(\Sigma_{\frac{m+1}{2}}-\nu(p t)\right) \times I$ (see figure 27). Note 


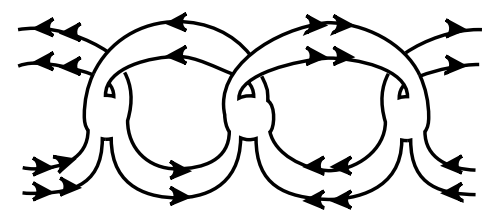

Figure 26: Fundamental domain for the 3:1 cover of $\left(\Sigma_{2}-\nu(p t)\right) \times I$

that the lift $\tilde{A}$ of the annulus $A$ is another annulus (enlarged from the original by a factor of $m$ ). Let $M_{1_{\tau}}^{+}$denote the $A_{+}$component of $M \backslash A$. We will establish tightness of $(\tilde{M} \backslash \tilde{A}, \tilde{\xi})$ where $\xi$ is the unique, non-product tight contact structure on $M \backslash A$ by focusing on the covering space $\left(\tilde{M} \backslash \tilde{A},\left.p\right|_{p^{-1}\left(M_{1}^{+}\right)}\right)$. The argument for the other component is completely analogous. Recall that the product structure is universally tight on $M_{1}$.

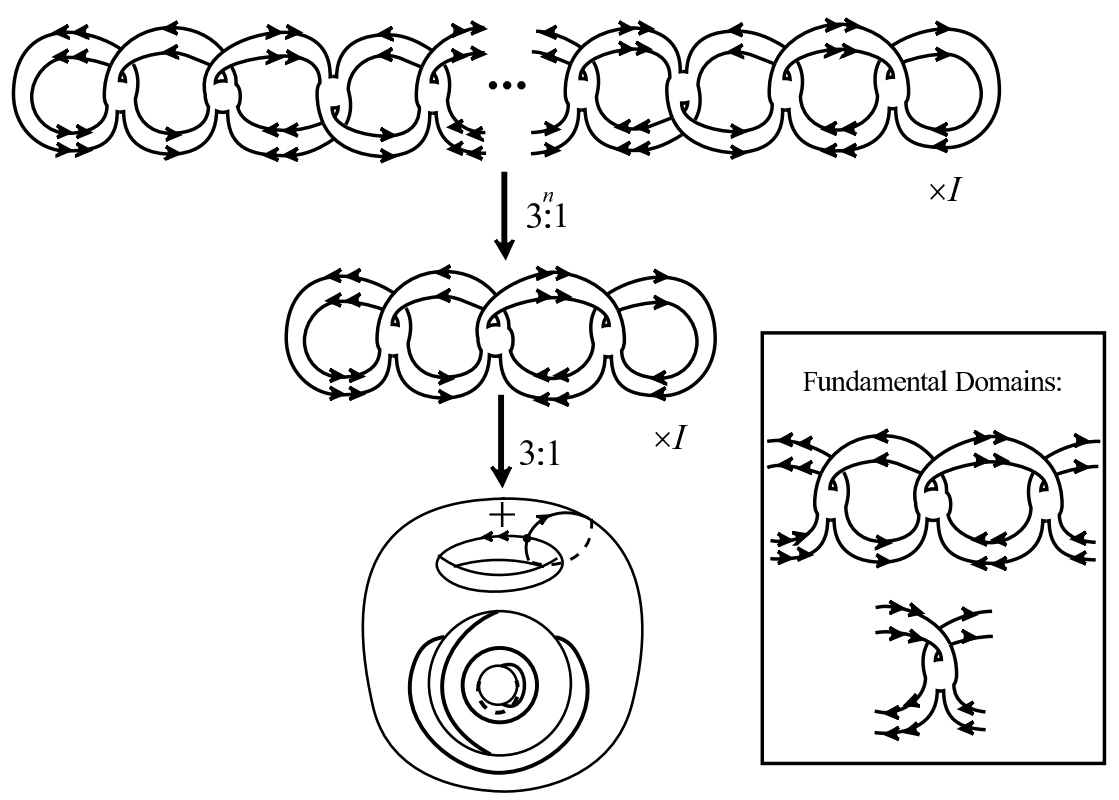

Figure 27: A covering space for $M_{1}$

Lemma 4.1.1 $(\tilde{M} \backslash \tilde{A}, \tilde{\xi})$ is a tight contact manifold where $\xi$ is the unique, non-product tight contact structure on $M \backslash A$.

Proof It suffices to consider $\left(\tilde{M} \backslash \tilde{A},\left.p\right|_{p^{-1}\left(M_{1}^{+}\right)}\right)$. Our aim is to prove tightness of $(\tilde{M} \backslash \tilde{A}, \tilde{\xi})$ by using Honda's gluing/classification theorem on the convex de- 
composition of $(\tilde{M} \backslash \tilde{A}, \tilde{\xi})$ which is the pullback of the one on $M \backslash A$ (see lemma 3.0.4 and figures [19] and 201. We will begin by lifting the cut on $M \backslash A$ which is transverse to the double-arrow direction as indicated for the 3:1 cover in figure 28. Recall that the $3: 1$ cover of $M_{1}^{+}$is $\Sigma_{2}-\nu(p t)$.
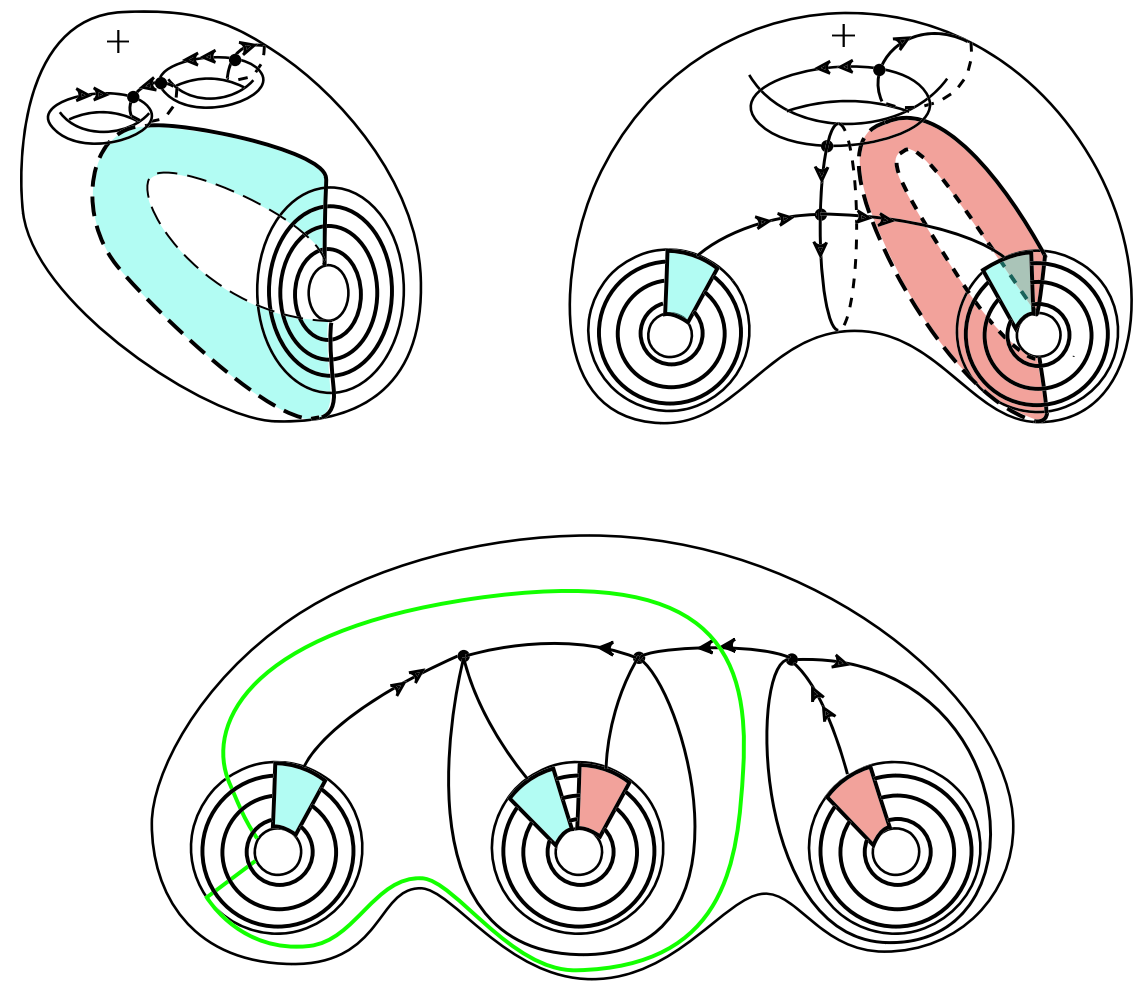

Figure 28: The pullback of the contact structure

Cutting open along the $m$ pull-backs of the first cutting disk downstairs yields $\frac{m-1}{2}+1$ solid tori. Our covering projection $p$ restricted to $\frac{m-1}{2}$ of these tori is a $2: 1$ cover, while $p$ restricted to the remaining torus is a $1: 1$ cover. These $\frac{m-1}{2}+1$ solid tori fall into three categories according to the way the cutting surfaces appear on them. One of the 2:1 covers always contains the positive and negative copies of one cutting surface, the positive copy of a second cutting surface, and the negative copy of a third. The 1:1 cover always contains the positive copy of a cutting surface and the negative copy of another. The remaining $\frac{m-1}{2}-1$ 2:1 covers contain copies of four different cutting surfaces, two positive and two negative. Examples of these three categories are given in figure 29 (note that no tori of the latter form appear in the decomposition of the 3:1 cover). 


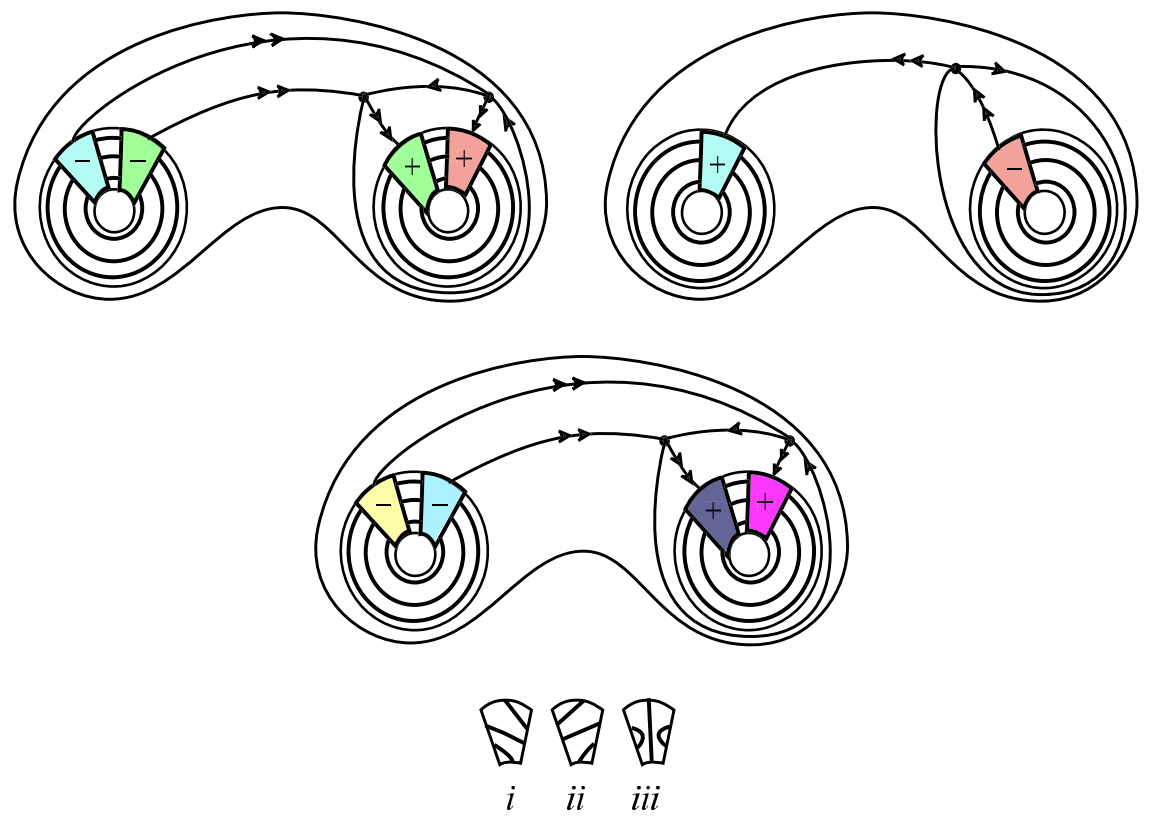

Figure 29: The pullback of the contact structure \#2

Now we begin pulling back the contact structure. At this stage, this means applying dividing curve configuration $i$ to the cutting surfaces (see lemma 3.0.4 and figure 18). The resulting boundary configurations (parallel sets of longitudinal dividing curves on the tori) are given for the $3: 1$ cover at the top of figure 30 along with three new convex cutting disks which are the pull-backs of the second cut downstairs (transverse to the single arrow direction). Pulling back the contact structure at this level means applying the dividing curve configuration of figure 19 (B) $i$ i to these disks. The result is $m$ 3-balls with a single dividing curve each.

This state is not obviously overtwisted (i.e. it is potentially allowable). Recall that the state transition $i$ to $i i$ along $\epsilon_{+}$exists downstairs while the state transition $i i$ to $i i i$ does not. The state transition $i$ to $i i$ downstairs corresponds to doing all such transitions along all lifts of $\epsilon_{+}$upstairs. It is necessary to check that doing any combination of these bypasses upstairs transitions us to a potentially allowable state. These state transitions can be checked explicitly and exist as trivial or folding bypasses on each torus. The results are dividing curve configurations on the tori consisting of either two, four or six parallel, longitudinal dividing curves. The non-existence of the state transitions $i i$ to $i i i$ and $i$ to $i i i$ downstairs does not imply the non-existence of any such state 

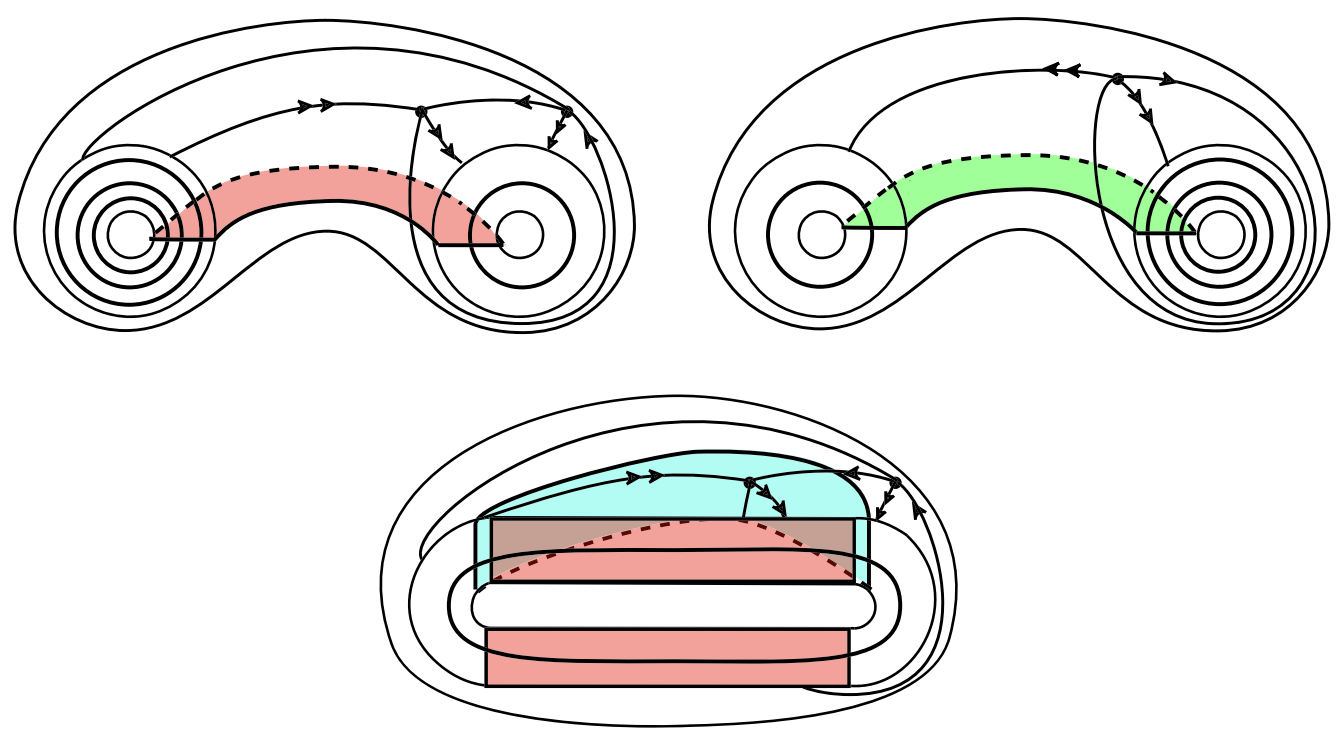

Figure 30: The pullback of the contact structure \#3

transition upstairs. However, it can be checked that this possibility never exists. The finiteness of the check is a result of the fact that, for any cover, the pullback decomposition results in the union of $m=3^{n}$ balls (each containing two copies of the pullback of each of the two cutting disks downstairs). These balls fall into three categories. Two of the $m$ balls contain two different types of self-gluing, while the remaining $m-2$ balls admit no self-gluing (see figure 31). Thus, we may conclude, by the gluing/classfication theorem, that $(\tilde{M} \backslash \tilde{A}, \tilde{\xi})$ is tight.

\subsection{An interpretation of the gluing theorem and tightness}

In order to establish tightness of the product structure we simply note that the pull-back $\tilde{\xi}$ of the product structure is a product structure, and this structure is tight by Giroux's criterion. For the potentially allowable non-product structure, we note that the proof of the standard gluing theorem (theorem 2.3.1 [16]) depends on two facts that we don't have:

(1) The dividing sets on cutting surfaces are boundary-parallel.

(2) The contact structure $\xi$ is universally tight on $M \backslash S$ where $S$ is our cutting surface. 


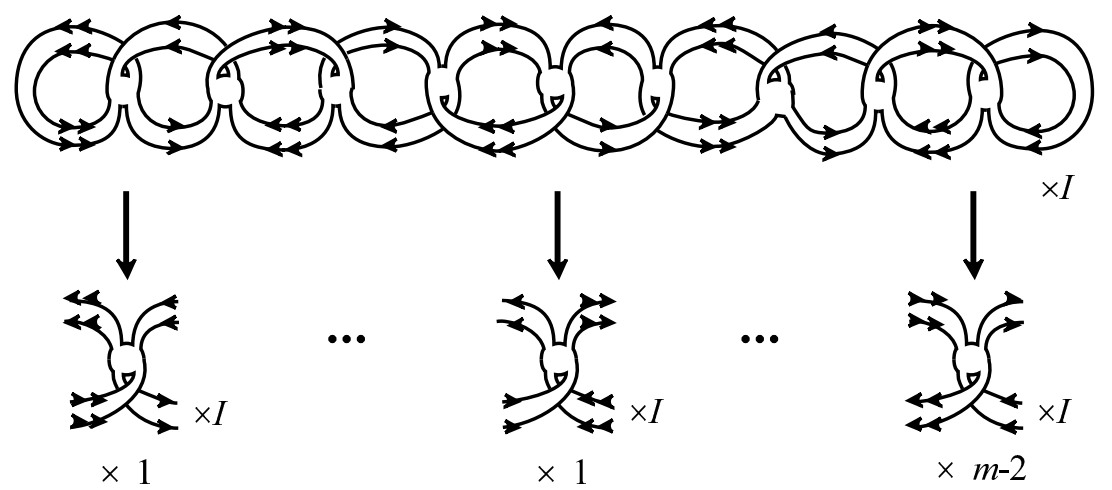

Figure 31: Decomposition of $\tilde{M}$ into three types of balls

However, we have classified our contact structures on $M \backslash A$ and know which bypasses are possible. By reviewing the proof of the gluing theorem, we see that the requirement that $\Gamma_{S}$ be boundary-parallel is there so that the types of bypasses possible along $S$ are strictly limited to those which are trivial or "long". In our case the types of bypasses possible along $A$ with $\Gamma_{A}=T 2_{1}^{+}$inside $(M, \xi)$ are similarly limited (where $\xi$ is the unique non-product structure). We have the following lemma.

Lemma 4.2.1 Let $A$ be a convex annulus whose boundary is as indicated in figure 8 with $\Gamma_{A}=T 2_{1}^{+}$inside the unique non-product $(M, \xi)$. Then, any convex annulus $A^{\prime}$ that is obtained from $A$ by a sequence of bypass moves will have a dividing set $\Gamma_{A^{\prime}}$ differing from $\Gamma_{A}$ by possibly adding an even number of parallel, closed dividing curves encircling the inner boundary component $\delta \times 1$ of $A$.

Proof Recall that, for the non-product structure on $M \backslash A$ with $\Gamma_{A}=T 2_{1}^{+}$, the bypasses indicated along $A_{+}$at the left of figure 17 and their counterparts along $A_{-}$do not exist. These bypasses also do not exist in any of the covers constructed in the previous section for similar reasons (see figure 30 and lemma 3.0.4). By examining all remaining possibilities, we see that the only bypasses that exist along $A$ in this case are trivial bypasses and folding bypasses. Further bypasses may be trivial (i.e. they produce no change in the dividing set), may add pairs of parallel dividing curves, or may delete pairs of dividing curves. Examples of these possibilities are given in figure 32. So, the dividing set on any annulus $A^{\prime}$ obtained from $A$ by a sequence of bypass moves has dividing set $\Gamma_{A^{\prime}}$ that differs from $A$ by adding, at most, pairs of homotopically non-trivial closed dividing curves parallel to the original one. 

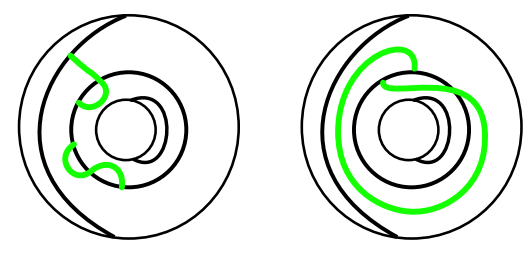

Figure 32: Some bypass possibilities

To address our second problem, let us consider the strategy of the of the proof of the gluing theorem. The proof is by contradiction. The existence of an overtwisted disk $D \subset M$ is supposed, and we consider the sequence of bypass moves required to isotope $A$ off of $D$. The requirement that $\xi$ is universally tight on $M \backslash A$ is so that, when analyzing a single bypass move along $A$ (which is trivial or increases $\# \Gamma_{A}$ ), we can lift to a large enough cover so as to make this bypass trivial. We continue to lift to covers until we arrive at the cover $\tilde{M}$ of $M$ with lifts $\tilde{A}$ of $A$ and a lift $\tilde{D}$ of a proposed overtwisted disk $D$ in which all bypasses needed to isotope $\tilde{A}$ off of $\tilde{D}$ (producing the isotoped annulus $\tilde{A}^{\prime}$ ) are trivial. But $\Gamma_{\tilde{A}^{\prime}}=\Gamma_{\tilde{A}}$ where $\tilde{A}^{\prime}$ is obtained from $\tilde{A}$ by a sequence of trivial bypass attachments. Since $\xi$ is universally tight on $M \backslash A$, this gives us a contradiction.

The objective of the previous section was to construct specific covers of $\tilde{M}$ so that $\tilde{M} \backslash \tilde{A}$ is tight. These covers suffice to satisfy the requirements of the gluing theorem. Thus, we are able to establish tightness of the potentially tight non-product $(M, \xi)$.

We repeat here two lemmas from the proof of the gluing theorem [16]. The first one concerns isotoping $A$ off of an overtwisted disk $D$. By a result of Honda [14, we may perturb the characteristic foliation on the cutting surface and look at the local model near points on the boundary of the disk. We can arrange for $D$ to be transverse to $A$ and for $\partial D$ to be contained in $\Gamma_{A}$. Moreover, after possibly modifying $D$, we can assume that the hypotheses of the Legendrian realization principle are satisfied so that $D \cap A$ consists of Legendrian arcs and curves. This is called the "controlled intersection" of an overtwisted disk with a convex cutting surface $A$.

Now, to push $A$ away from $D$ so that we eliminate a closed curve of intersection $\delta$. Let $D_{\delta}$ denote the subdisk of $D$ with $\partial D_{\delta}=\delta$. Since we assume $A$ has a tight neighborhood, we must have that $t\left(\delta, F r_{A}\right)<0$. We may perturb $D_{\delta}$ (rel boundary) so as to make it convex with Legendrian boundary.

We have that $\stackrel{\circ}{D}_{\delta}$ is contained in $M \backslash A$, which is tight. So we may assume that the dividing set of $D_{\delta}$ consists only of embedded arcs with endpoints on 
$\partial D$. We may push $A$ to engulf the bypass which corresponds to one of these boundary-parallel dividing curves on $D_{\delta}$. We continue until all bypasses are consumed.

For an arc of intersection $\gamma$ in $D \cap A$, we proceed similarly, but we choose a disk $D_{\gamma}$ with $\gamma \subset \partial D_{\gamma}$. This concludes the sketch of the following lemma:

Lemma 4.2.2 It is possible to isotope $A$ off of $D$ in a finite number of steps, each of which is a bypass along $A$.

The second lemma concerns the effect of isotoping cutting surfaces across trivial bypasses. A trivial bypass along a convex surface $A$ can be realized inside an $I$-invariant ("contact product") neighborhood of the surface.

Lemma 4.2.3 If $A$ is a convex surface with Legendrian boundary inside a contact manifold $(M, \xi)$ and $A^{\prime}$ is a convex surface obtained from $A$ by a trivial bypass, then $A$ and $A^{\prime}$ are contact isotopic and, hence, $(M \backslash A, \xi)$ is tight if and only if $\left(M \backslash A^{\prime}, \xi\right)$ is tight.

We are now ready to prove an adaption of the gluing theorem. Much of this proof is identical to the general proof.

Theorem 4.2.4 The potentially tight non-product structure is tight on $M$.

Proof Assume that $M$ is not tight. Then, there exists an overtwisted disk $D \subset$ $M$. We can perform a contact isotopy so that $D$ and $A$ intersect transversely along Legendrian curves and arcs and so that $\partial D \cap A \subset \Gamma_{A}$ 14. We note that closed curves in $D \cap A$ are homotopically trivial in $A$. We want to eliminate the innermost closed curves on $D$ by pushing $A$ across $D$. Consider a twosphere $S$ formed by a disk on $D$ and one on $A$ whose common boundary is an innermost curve of intersection $\delta \subset D$. Then, $S$ bounds a ball across which we can isotope A.

By lemma 4.2.2 we can push $A$ across $D$ to decrease the number of intersections of $A$ with $D$ in a finite number of bypass steps that possibly change the dividing curve configuration on the isotoped annulus. If we consider a single bypass along $A$, we know it is either trivial or increases $\Gamma_{A}$ (see the proof of lemma 4.2.1). Recall that the covering spaces constructed in the last section enlarge $A$ by a factor of $m$. So we can lift to a large enough cover of this type so that any folding bypass attachment becomes trivial. We can continue lifting through covers of this type until we arrive at the cover $\tilde{M}$ of $M$ with lifts $\tilde{A}$ of $A$ and 
$\tilde{D}$ of a proposed overtwisted disk $D$ in which all bypasses needed to isotope $\tilde{A}$ off of $\tilde{D}$ are trivial. But $\Gamma_{\tilde{A}^{\prime}}=\Gamma_{\tilde{A}}$ where $\tilde{A}^{\prime}$ has $\tilde{A}^{\prime} \cap \tilde{D}=\emptyset$ and is obtained from $\tilde{A}$ by a sequence of trivial bypass attachments. Since $\tilde{\xi}$ is tight on $M \backslash \tilde{A}$, then, by lemma 4.2.3 $\tilde{\xi}$ is tight on $M \backslash \tilde{A}^{\prime}$. This contradicts the existence of an overtwisted disk in $M \backslash \tilde{A}^{\prime}$. Thus, $(M, \xi)$ is tight.

\section{A special property of the non-product tight con- tact structure on $M$}

By viewing the non-product tight contact structure on $M=\Sigma \times I$ from the perspective of a different convex decomposition, it is possible to see that this contact structure contains every possible bypass abutting the boundary.

We perform a second convex decomposition assuming we are starting with the non-product structure $\xi$ on $M$. The first cut of this decomposition will be along $\delta \times I$ where $\delta \times\{i\}$ is a homotopically nontrivial non-separating curve which is efficient with respect to $\Gamma_{\Sigma \times\{i\}}, i \in[0,1]$ (see figure 33). After this initial annular cut, we have $M_{1}=M \backslash A$ which is a twice punctured torus cross $I$ as in figure 34. We have the same four possibilities for $\Gamma_{A}$ as in the previous decomposition (see figure 9), but in this case it is easy to see that all $T 2_{2 m}^{ \pm}$, $m \in \mathbb{Z}^{+}$are overtwisted. We have the following lemmas. The proofs will be omitted since they are similar to the proofs of the reduction lemmas for the previous decomposition.

(1) Lemma: $T 2_{2 m+1}^{ \pm}, m \in \mathbb{Z}^{+}$can be reduced to $T 2_{1}^{ \pm}$.

(2) Lemma: $T 1_{k}, k \in \mathbb{Z}^{+}$can be reduced to $T 1_{1}$.

(3) Lemma: $T 1_{k}, k \in \mathbb{Z}^{-}$can be reduced to $T 1_{-1}$.

Since we have already shown that the non-product structure is tight, we will focus our attention on showing that the non-product structure, viewed from the perspective of the current decomposition, has a cut of type $T 2_{1}^{+}$and one of type $T 2_{1}^{-}$. We will do this by showing that there is a non-product contact structure in each of the categories $T 2_{1}^{+}, T 2_{1}^{-}, T 1_{1}$ and $T 1_{-1}$ and we can find isotopies transforming each of these structures into one-another.

Theorem 5.0.5 There exists exactly one universally tight contact structure on $M_{1}$, not equivalent to $T 1_{0}$, in each of the categories $T 1_{ \pm 1}$ and $T 2_{1}^{ \pm}$. 


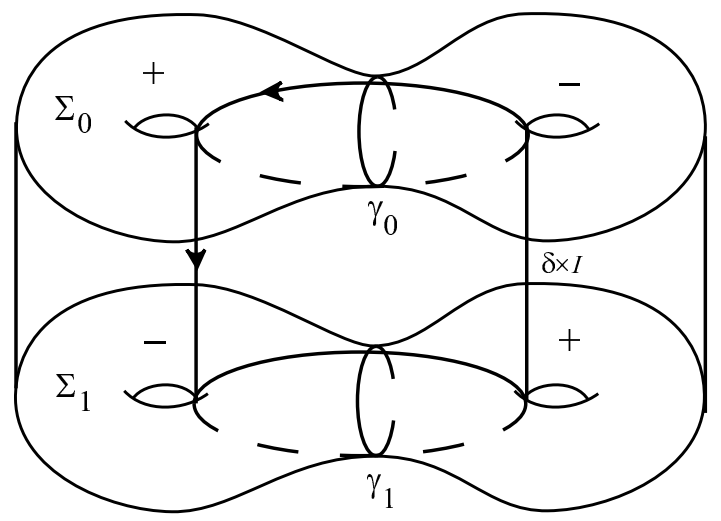

Figure 33: $\mathrm{M}=\Sigma_{2} \times I$

Proof We show that there is exactly one non-product tight contact structure on $M_{1}$ with $\Gamma_{A}$ of type $T 1_{1}$. To do this, we provide a convex decomposition of $M=\Sigma \times I$ starting with the convex annulus $A$ together with $\Gamma_{A}=T 1_{1}$. The convex disks defining this decomposition are given in figures 35 and 36. The other cases are argued similarly.

Round edges along $\partial A_{ \pm} \subset M_{1}$ and choose a new cutting surface $\delta \times I$ where $\delta$ is indicated in figure 35(B). Assume $\delta \times I$ is convex with efficient Legendrian boundary. After cutting along $\delta$, we have a new manifold $M_{2}$ which is a punctured torus cross $I$ (see figure 35 (D)). Two copies of the cutting surface $\delta \times I$ appear in $M_{2}$ that we will call $\epsilon_{+}$and $\epsilon_{-}$. Since $t b\left(\partial \epsilon_{ \pm}\right)=-2$, there exist

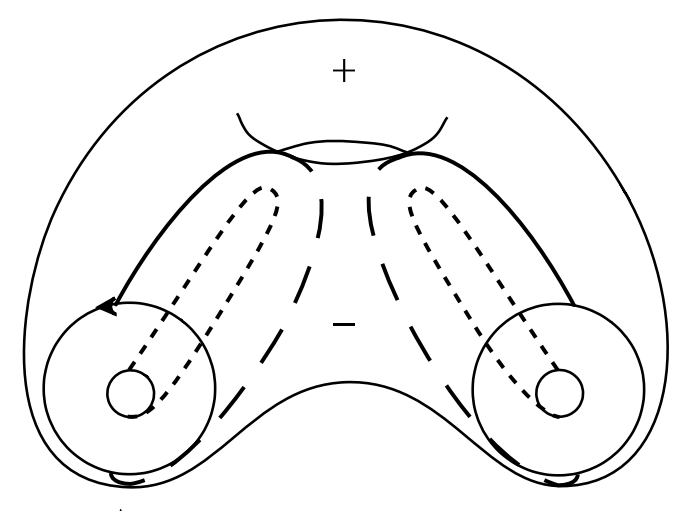

$\mathrm{A}_{+}$

$\mathrm{A}-$

Figure 34: $M_{1}=M \backslash A$ 


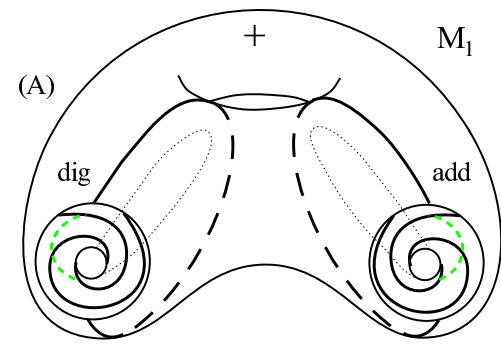

$\mathrm{A}_{+}$

A_

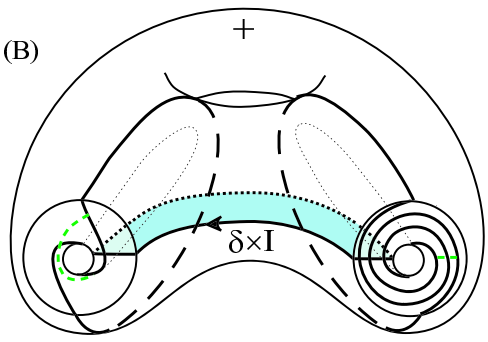

(C)
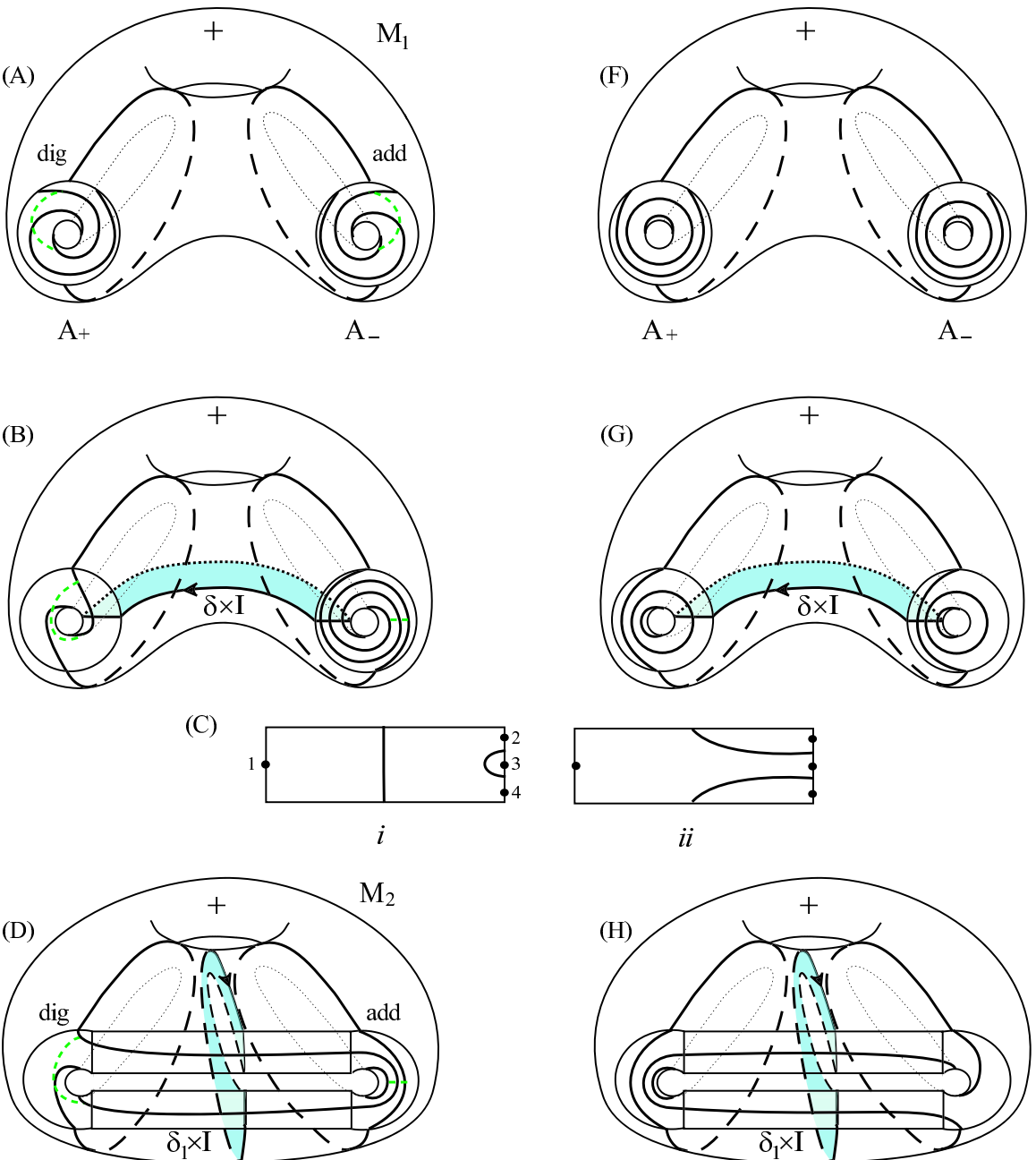

$\mathrm{A}_{+}$

A-
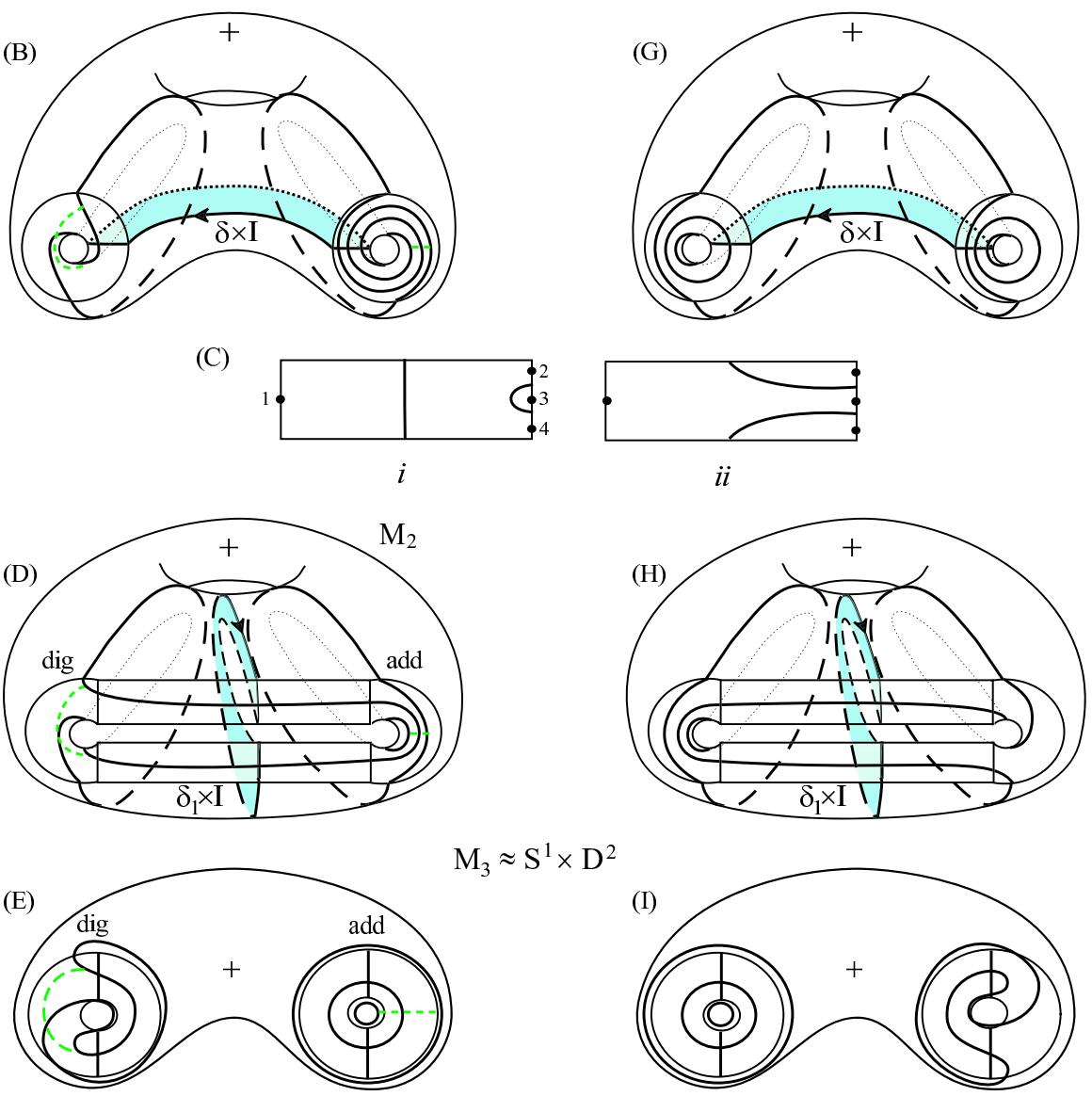

$\mathrm{M}_{3} \approx \mathrm{S}^{1} \times \mathrm{D}^{2}$

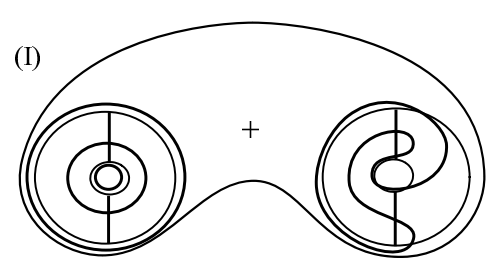

Figure 35: Convex decomposition \#1 for $T 1_{1}$ and $T 2_{1}^{+}$(1)

two possible dividing curve configurations for $\Gamma_{\epsilon_{+}}$. One of these configurations induces a bypass half-disk straddling position 3 as shown in figure [35 (C) $i$. 
Isotoping $A_{-} \subset M_{1}$ across this bypass produces a new dividing set on the isotoped annulus $A_{-}^{\prime}$ equivalent to $T 1_{0}$.

Let $\Gamma_{\epsilon_{+}}$be the remaining choice. After rounding edges along $\partial \epsilon_{ \pm}$, we obtain $\left(M_{2}, \Gamma_{\partial M_{2}}\right)$ as in figure 35 (D). After choosing a new convex cutting surface $\delta_{1} \times I$ with efficient Legendrian boundary as in figure 35] (D), we cut open $M_{2}$ and obtain $\left(M_{3} \cong S^{1} \times D^{2}, \Gamma_{\partial M_{2}}\right)$ with two copies of the cutting surface $\delta_{1} \times I$. Call these copies $\tau_{+}$and $\tau_{-}$. Since $t b\left(\partial \tau_{ \pm}\right)=-1$, there is exactly one possibility for $\Gamma_{\tau_{+}}$. Applying this configuration to $\tau_{+}$and $\tau_{-}$and rounding edges along $\partial \tau_{ \pm}$leads to the configuration $\left(M_{3}, \Gamma_{\partial M_{3}}\right)$ as shown in figure 35 (E).

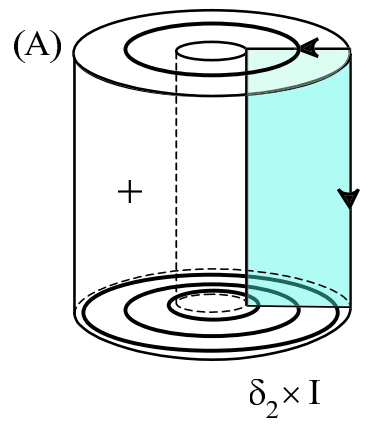

(B)

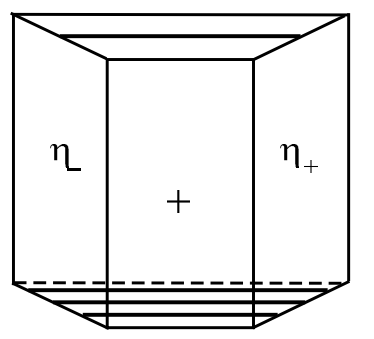

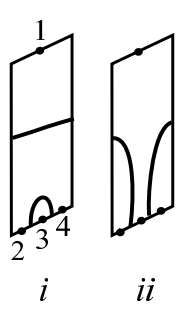

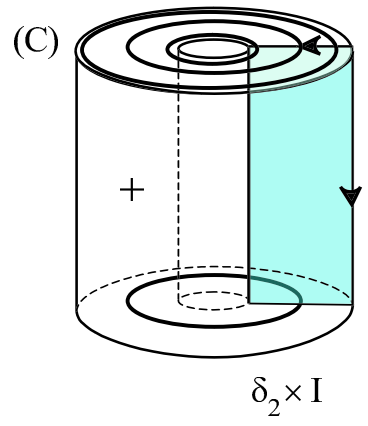

$\mathrm{M}_{4} \approx \mathrm{B}^{3}$

(D)

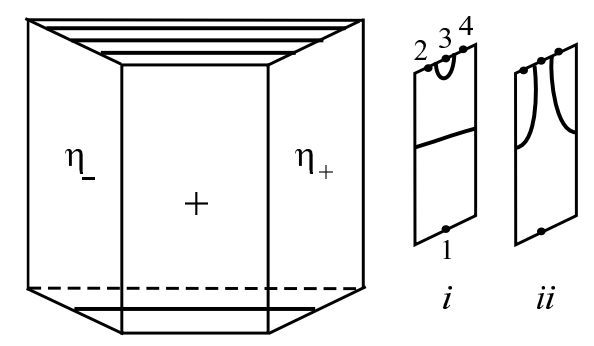

Figure 36: Convex decomposition \#1 for $T 1_{1}$ and $T 2_{1}^{+}(2)$

We view $M_{3}$ as a solid torus and choose a convex, meridional cutting surface $\delta_{2} \times I$ with efficient Legendrian boundary as in figure 36(A). We cut open $M_{2}$ along this surface to obtain $\left(M_{4} \cong B^{3}, \Gamma_{\partial M_{2}}\right)$ with two copies of the cutting surface $\delta_{1} \times I$. Call these copies $\eta_{+}$and $\eta_{-}$. Since $t b\left(\partial \eta_{ \pm}\right)=-2$, there are two possibilities for $\Gamma_{\eta_{+}}$. One of these configurations induces a bypass half-disk straddling position 3 as shown in figure 36 (B) $i$. Isotoping $A_{-} \subset M_{1}$ across this bypass produces a new dividing set on the isotoped annulus $A_{-}^{\prime}$ equivalent to $T 1_{0}$. 
Let $\Gamma_{\eta_{+}}$be the remaining choice. After rounding edges along $\partial \eta_{ \pm}$, we obtain $M_{4} \cong B^{3}$ with $\#\left(\Gamma_{\partial M_{4}}\right)=1$. Thus there is at most one non-product tight contact structure on $M_{1}$ with $\Gamma_{A}$ of type $T 1_{1}$.

Now, by Eliashberg's uniqueness theorem, there is a unique universally tight contact structure on $M_{4}=B^{3}$ which extends the one on the boundary. Also, the dividing sets on the convex disks $\epsilon, \tau$ and $\eta$ corresponding to our unique non-product contact structure were all boundary parallel. Therefore, by the gluing theorem [2, 17, there is a unique, universally tight contact structure on $M_{1}$ with $\Gamma_{A}$ of type $T 1_{1}$. Similarly, we can establish the existence of a such a structure structure on $M_{1}$ with $\Gamma_{A}$ of types $T 2_{1}^{+}$(given in figures 35] (F) through (I) and (C) and 36(C) and (D)), T2- (figure 37 (A) through (D)) and $T 1_{-1}$ (figure $37(\mathrm{E})$ through $(\mathrm{G})$ and $(\mathrm{C})$ ).

Lemma 5.0.6 The unique, non-product universally tight contact structures on $M_{1}$ of type $T 1_{ \pm 1}$ and $T 2_{1}^{ \pm}$are all equivalent.

Proof We prove the equivalences $T 1_{1} \cong T 2_{1}^{+}$. The others are done similarly. We start by taking a convex decomposition of $M=\Sigma \times I$ starting with the convex annulus $A$ together with $\Gamma_{A}=T 1_{1}$. The convex disks defining this decomposition are given in figures 35 and 38 . The purpose is to find a bypass half disk $B$ as indicated in figure 35 (A) so that digging $B$ from one side of $A$ and adding it to the other transforms $\Gamma_{A}=T 1_{1}$ into $\Gamma_{A}=T 2_{1}^{+}$. The other cases are argued similarly.

First, consider the partial convex decomposition $M_{3}=M_{1} \backslash(\epsilon \cup \tau)$ of the unique non-product $T 1_{1}$ as in the previous lemma and figures 35 (A) (B) (C) (D) and (E). We now proceed with a slightly different decomposition. View $M_{3}$ as a solid torus and choose a convex, meridional cutting surface $\delta_{2} \times I$ with Legendrian boundary. In order to prove existence of the proposed bypass half-disk $B$, our choice of cutting surface shown in figure 38 (A) is not efficient.

We observe that the proposed bypass is a folding bypass, and such bypasses always exist [16]. However, it is easy to show existence explicitly in our case by proceeding with the decomposition.

After cutting $M_{3}$ open along $\delta_{2} \times I$, we obtain the ball $\left(M_{4}, \Gamma_{\partial M_{3}}\right)$. Two copies of the cutting surface $\delta_{2} \times I$ appear in $M_{4}$. Call them $\eta_{+}$and $\eta_{-}$. There exist five choices for $\Gamma_{\eta_{+}}$as pictured in figure 38 (C). Applying choice $i$ or $i i$ of $\Gamma_{\eta_{+}}$ and rounding edges along $\partial \eta_{ \pm}$yields a dividing set on $\partial M_{4} \approx S^{2}$ consisting of three dividing curves. By Giroux's criterion, we conclude the existence of 
(A)

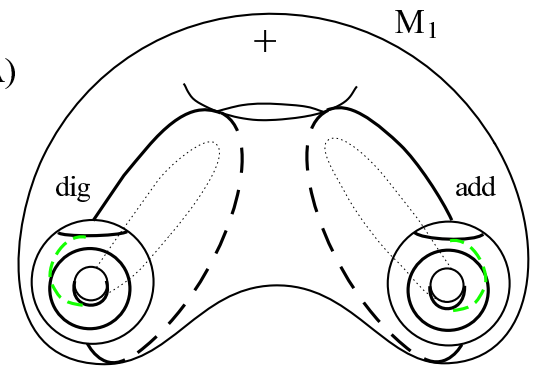

$\mathrm{A}+$

(B)

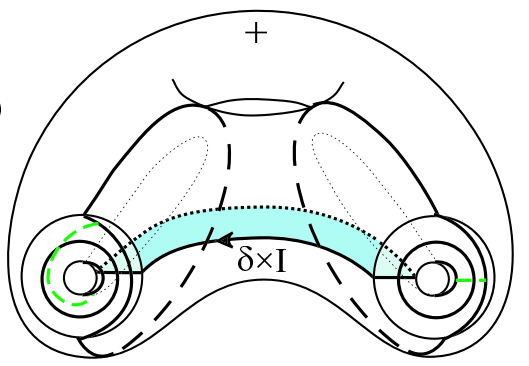

(C)

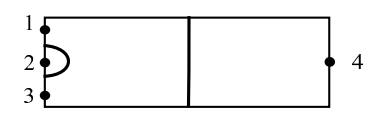

(D)

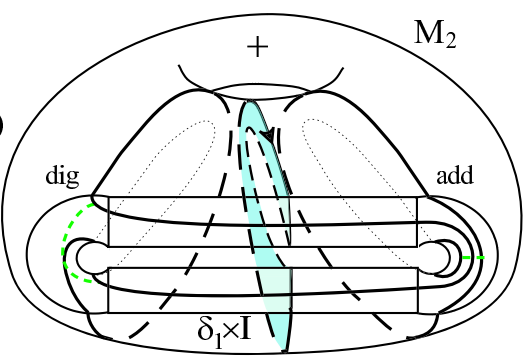

(E)

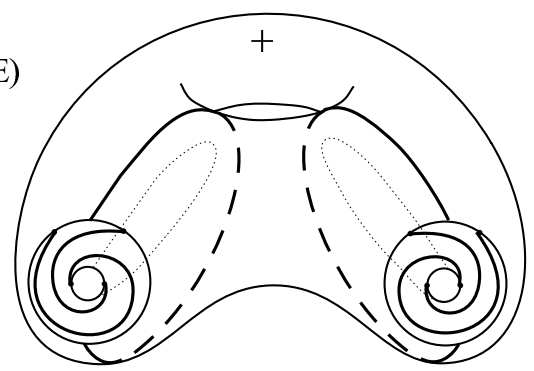

$\mathrm{A}_{+}$

A_

(F)

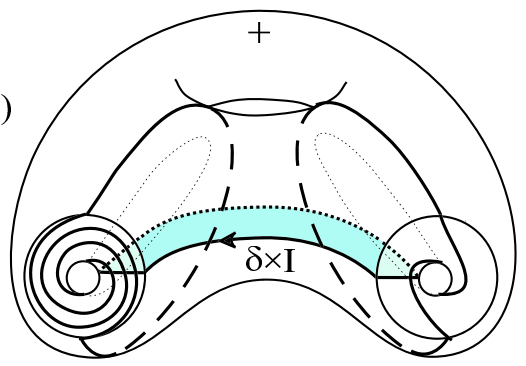

(G)

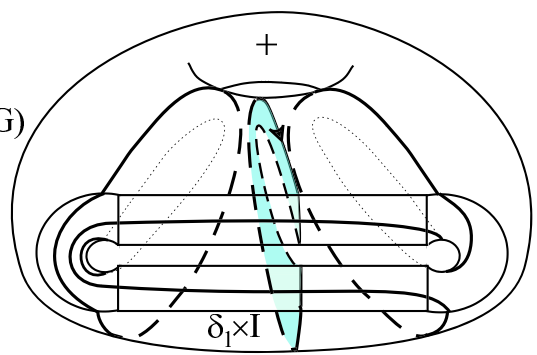

Figure 37: Convex decomposition \# 1 for $T 2_{1}^{-}$and $T 1_{-1}$

an overtwisted disk. Applying choice $i i i$ of $\Gamma_{\eta_{+}}$induces a bypass half-disk straddling position 5. Isotoping $A_{-} \subset M_{1}$ across this bypass produces a new dividing set on the isotoped annulus $A_{-}^{\prime}$ equivalent to $T 1_{0}$.

Both choices $i v$ and $v$ correspond to a dividing curve configuration on $S^{2} \approx \partial B^{3}$ such that $\# \Gamma_{\partial B^{3}}=1\left(\Gamma_{\partial B^{3}}\right.$ resulting choice $i v$ is illustrated in figure $\left.38(\mathrm{D})\right)$. We would like to find a state transition taking dividing curve configuration iv to dividing curve configuration $v$. To do this, we must establish the existence 
(A)

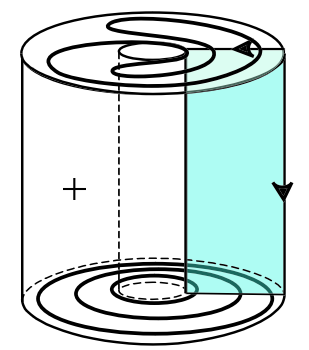

(B)

$$
\delta_{2} \times \mathrm{I}
$$

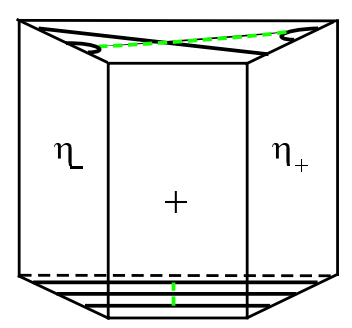

(C)

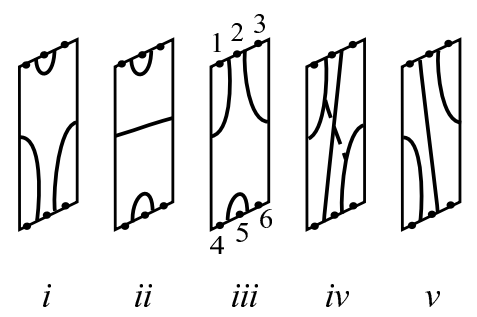

(D)

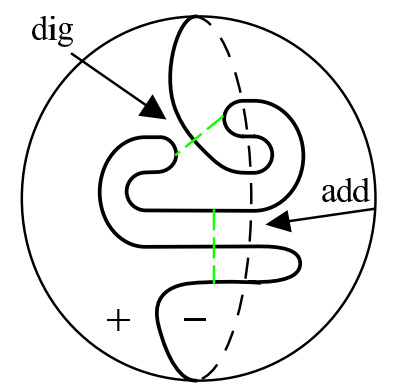

(E)

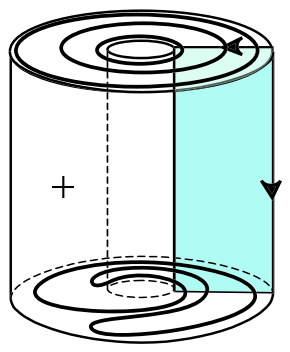

(F)

$\delta_{2} \times \mathrm{I}$

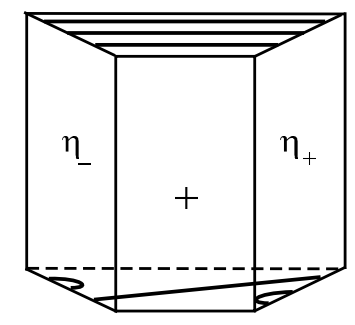

(G)

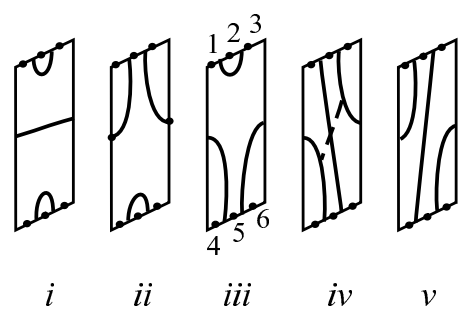

Figure 38: Equivalence of $T 1_{1}$ and $T 2_{1}^{+}$ 
of a bypass half-disk as indicated by it's attaching arc in choice $i v$ of figure 38 (C). Applying choice $i v$ to $\partial M_{4} \approx S^{2}$, we see that this bypass is the trivial one on the ball. Such a bypass is guaranteed to exist by the right to life principle [17. Thus, we have shown the existence the proposed bypass $B$ taking $T 1_{1}$ to $T 2_{1}^{+}$.

We proceed by performing the same convex decomposition on $M_{1}$ with $\Gamma_{A}$ of type $T 2_{1}^{+}$. We then argue that digging $B$ yields a state transition transforming the unique potentially non-product $T 1_{1}$ into the unique potentially non-product $T 2_{1}^{+}$. The convex disks defining this decomposition are given in figures 35 (F) $(\mathrm{G})(\mathrm{C})(\mathrm{H})$ and $(\mathrm{I})$ and $38(\mathrm{E})(\mathrm{F})$ and $(\mathrm{G})$.

We proceed exactly as in the previous case, noting that, in the third stage of the decomposition of $M_{1}$, the boundary of our cutting surface $\eta$ shown in figure 38 (E) is not efficient. Since $t b\left(\partial \eta_{ \pm}\right)=-3$, we will have five possibilities for $\Gamma_{\eta_{+}}$. Cases $i$ and $i i$ in figure $38(\mathrm{G})$ lead to $\#\left(\Gamma_{\partial B^{3}}\right)=3$, and case $i i i$ induces a bypass that can be realized on $A_{-} \subset M_{1}$ transforming $T 2_{1}^{+}$into $T 1_{0}$. Finally, there exists a state transition, as indicated in figure $38(\mathrm{G}) i v$ taking case $i v$ to case $v$.

We will now show that, at each stage of the convex decomposition, digging the bypass along a section of $A_{+}$and adding it back along a section of $A_{-}$transforms the unique non-product $T 1_{1}$ into the unique non-product $T 2_{1}^{+}$. First, consider $M_{1}=M \backslash A$ with $\Gamma_{A}=T 1_{1}$ as pictured in figure 35 (A). If we dig the bypass along $A_{+}$and add it back along $A_{-}$as indicated, the result is exactly $M_{1}=M \backslash A$ with $\Gamma_{A}=T 2_{1}^{+}$as pictured in figure 35 (F). Second, consider $M_{2}=M_{1} \backslash(\delta \times I)$ as pictured in figure 35 (D). If we dig the bypass along $A_{+}$ and add it back along $A_{-}$as indicated, the result is exactly $M_{2}=M_{1} \backslash(\delta \times I)$ with $\Gamma_{A}=T 2_{1}^{+}$as pictured in figure $35(\mathrm{H})$. Third, consider $M_{3}=M_{2} \backslash\left(\delta_{1} \times I\right)$ as pictured in figure 35(E). If we dig the bypass along $A_{+}$and add it back along $A_{-}$as indicated, the result is exactly $M_{3}=M_{2} \backslash\left(\delta_{1} \times I\right)$ with $\Gamma_{A}=T 2_{1}^{+}$ as pictured in figure 35(I). Finally, consider $M_{4}=M_{3} \backslash\left(\delta_{2} \times I\right)$ as pictured in figure 38 (D). If we dig the bypass and add it back as indicated, the result is exactly $M_{4}=M_{3} \backslash\left(\delta_{2} \times I\right)$ with $\Gamma_{A}=T 2_{1}^{+}$with $\#\left(\Gamma_{\partial M_{4}}\right)=1$. Thus, we have that the unique non-product $T 1_{1}$ is equivalent to the unique non-product $T 2_{1}^{+}$.

To obtain the equivalence $T 2_{1}^{-} \cong T 1_{-1}$, we use the same convex decomposition as in the previous cases. For the case $M_{1}$ with $\Gamma_{A}=T 2_{1}^{-}$and $T 1_{-1}$, the convex decomposition is given partly in figure 37 The rest of the decomposition is as in figure 39

For the equivalences $T 1_{1} \cong T 2_{1}^{-}$and $T 2_{1}^{+} \cong T 1_{-1}$, we use a different convex decomposition to avoid cutting through the proposed bypasses. The convex 
(A)

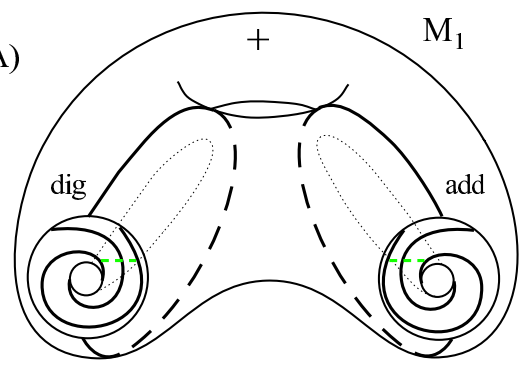

$\mathrm{A}_{+}$

A

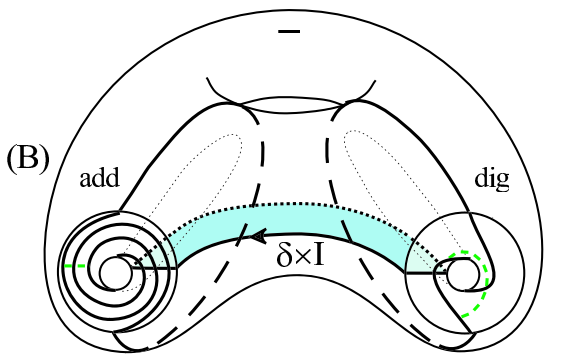

(E)

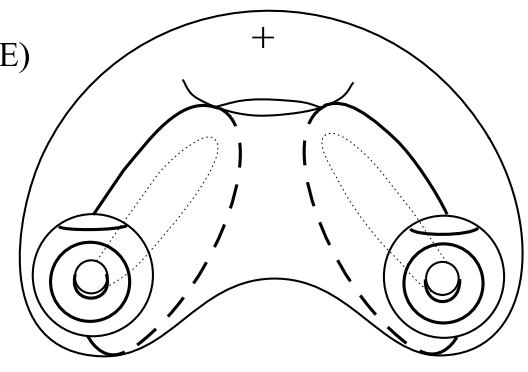

$\mathrm{A}_{+}$

A_

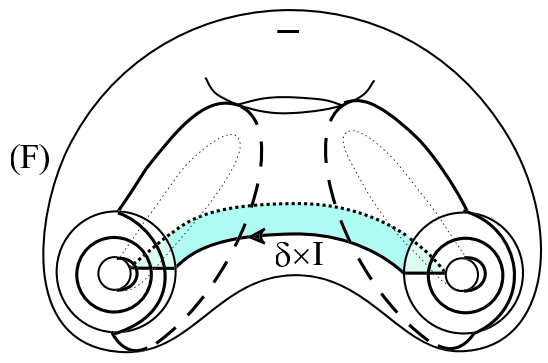

(C)
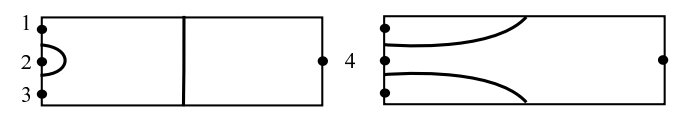

(D)
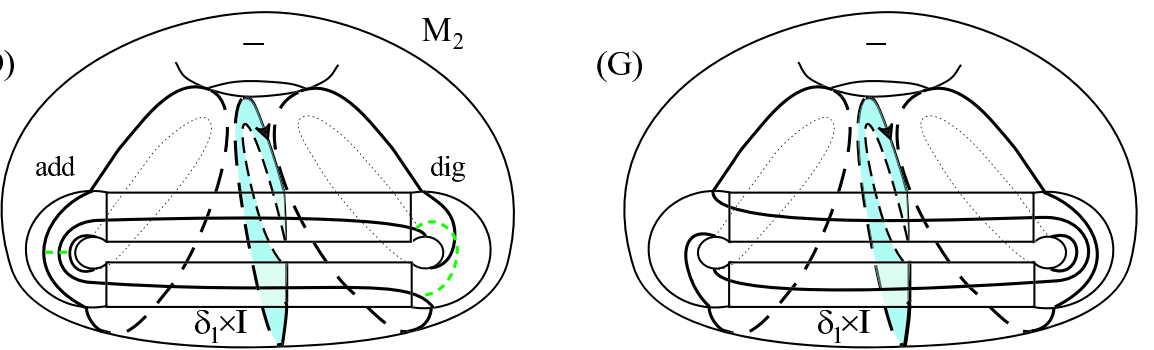

Figure 39: Equivalence of $T 1_{1}$ and $T 2_{1}^{-}$

(1)

decomposition for the first of these two transitions is given in figures 39 and 40 . For the equivalence $T 2_{1}^{+} \cong T 1_{-1}$, the convex decomposition is given partly in figure 41. The rest of the decomposition is as in figure 40.

By our previous classification, we may conclude that the non-product structure we have found is the unique non-product tight contact structure on $M$. Moreover, this decomposition shows us that the non-product structure has a cut of 


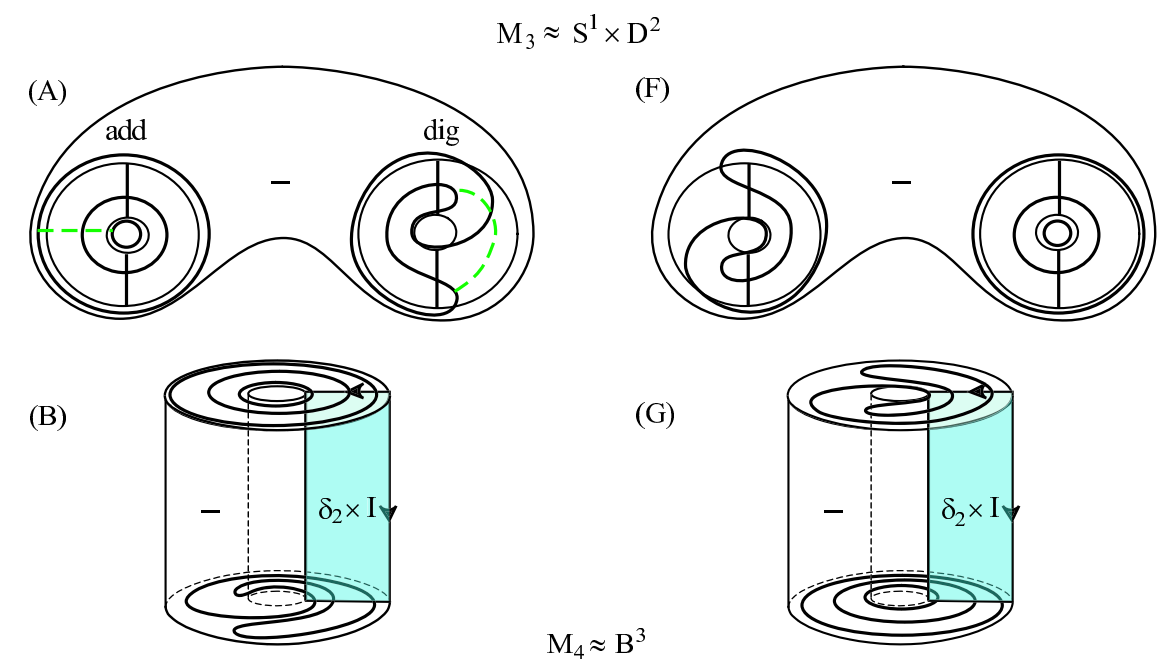

(C)

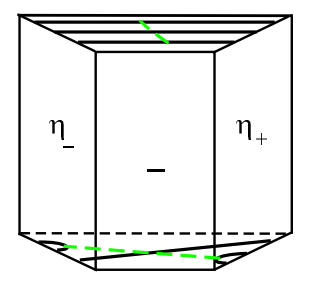

(D)

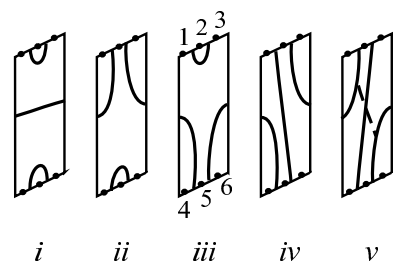

(E)

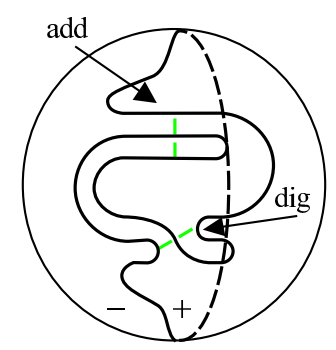

(H)

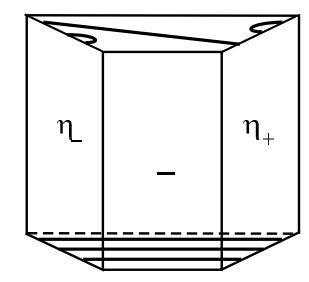

(I)

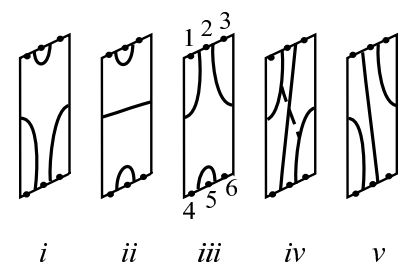

Figure 40: Equivalence of $T 1_{1}$ and $T 2_{1}^{-}(2)$ 
(A)

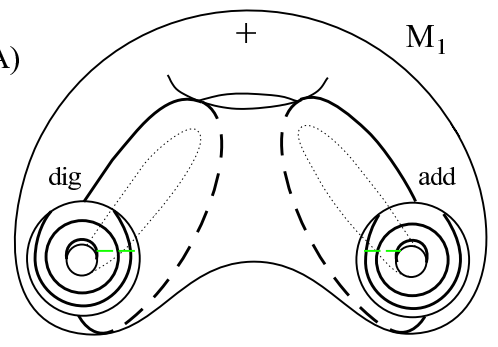

$\mathrm{A}_{+}$

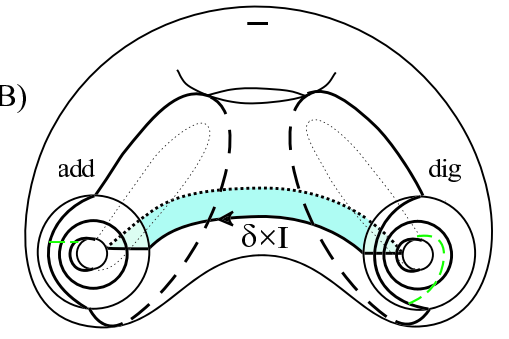

(C)

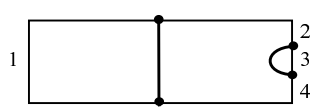

(D)

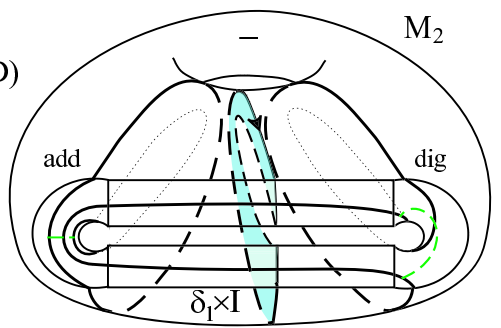

(E)

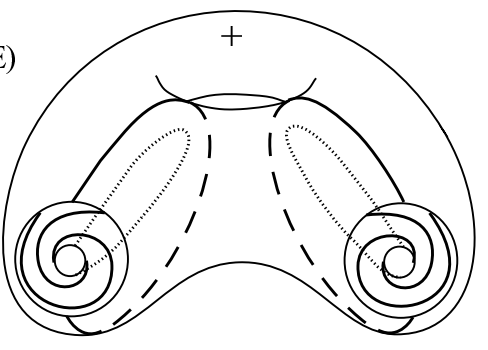

$\mathrm{A}_{+}$

A-

(F)
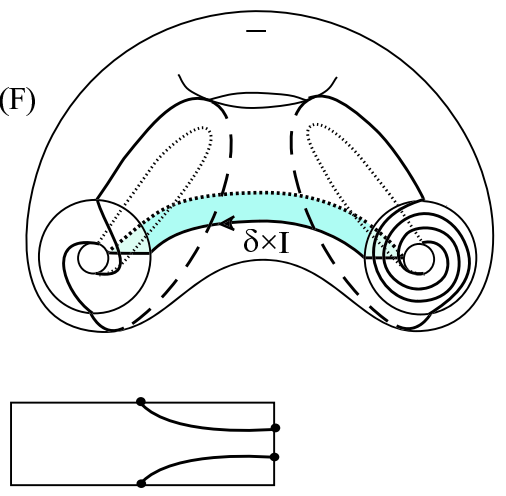

(G)

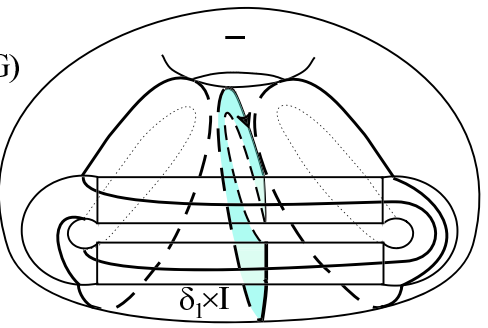

Figure 41: Equivalence of $T 2_{1}^{+}$and $T 1_{-1}$

type $T 2_{1}^{+}$and one of type $T 2_{1}^{-}$. So, if either boundary component, $\delta \times\{0\}$ or $\delta \times\{1\}$, of our initial annular cutting surface is the attaching arc of of a bypass, then our manifold contains all possible complementary bypasses. Thus, this attachment would result in an overtwisted structure.

We can find a diffeomorphism of the surface, fixing the dividing set, taking this $\delta$ to any other curve that can be the attaching arc of a non-trivial bypass, and this diffeomorphism may be extended to the product. Since there is 
unique non-product structure, this must take the non-product structure $\xi$ to the non-product structure, and, hence, all bypasses must exist along the image of $\delta$. It follows that this manifold contains every allowable bypass abutting the boundary. This means that if $(M, \xi)$ is contained in some tight $\left(M^{\prime}, \xi^{\prime}\right)$, then for any convex surface with boundary $S \subset M^{\prime}$ such that $\partial S \subset \partial M$ and $\#\left(\partial S \cap \Gamma_{\partial M}\right)=2$, the dividing set on $S$ cannot contain any boundary-parallel dividing arcs. We may conclude that the neighborhood of this cutting surface $\Sigma_{2}$ inside a tight $\left(M^{\prime}, \xi^{\prime}\right)$ must be a product.

Acknowledgements I would like to thank my doctoral advisor, Gordana Matić, for her guidance and helpful comments. I would also like to thank Joan Birman for inspiring me and for providing indispensable advice and direction. My sincere gratitude goes to Nancy Wrinkle for her unflagging support, encouragement, and assistance throughout the entire process. I offer my gratitude also to the referee, whose detailed and thoughtful comments dramatically improved this paper. Finally, I would like to thank Will Kazez, Ko Honda, Clint McCrory, John Hollingsworth as well as numerous others for their significant contributions to my education.

\section{References}

[1] D Bennequin, Entrelacements et équations de Pfaff, Astérisque 107-108 (1983) 87-161 MathReview

[2] V Colin, Recollement de variétés de contact tendues, Bull. Soc. Math. France 127 (1999) 43-69 MathReview

[3] V Colin, Une infinité de structures de contact tendues sur les variétés toroiodales, Comment. Math. Helv. 76 (2001) 353-372 MathReview

[4] V Colin, E Giroux, K Honda, On the coarse classification of tight contact structures, from: "Topology and geometry of manifolds (Athens, GA, 2001)", Proc. Sympos. Pure Math. 71, Amer. Math. Soc. (2003) MathReview

[5] Y Eliashberg, Classification of overtwisted contact structures on 3-manifolds, Invent. Math. 98 (1989) 623-637 MathReview

[6] Y Eliashberg, Contact 3-manifolds twenty years since J. Martinet's work, Ann. Inst. Fourier (Grenoble) 42 (1992) 165-192 MathReview

[7] J Etnyre, Tight contact structures on lens spaces, Commun. Contemp. Math. 2 (2000) 559-577 MathReview

[8] J Etnyre, K Honda, On the nonexistence of tight contact structures, Ann. of Math. 153 (2001) 749-766 MathReview 
[9] E Giroux, Convexité en topologie de contact, Comment. Math. Helv. 66 (1991) 637-677 MathReview

[10] E Giroux, Structures de contact en dimension trois et bifurcations des feuilletages de surfaces, Invent. Math. 141 (2000) 615-689 MathReview

[11] E Giroux, Structures de contact sur les variétés fibrées en cercles au-dessus d'une surface, Comment. Math. Helv. 76 (2001) 218-262 MathReview

[12] K Honda, On the classifiction of tight contact structures I, Geom. Topol. 4 (2000) 309-368 MathReview

[13] K Honda, On the classifiction of tight contact structures II, J. Differential Geom. 55 (2000) 83-143 MathReview

[14] K Honda, Gluing tight contact structures, Duke Math. J. 115 (2002) 435-478 MathReview

[15] K Honda, W Kazez, G Matić, Tight contact structures and taut foliations, Geom. Topol. 4 (2000) 219-242 MathReview

[16] K Honda, W Kazez, G Matić, Convex decomposition theory, Internat. Math. Res. Notices (2002) 55-88 MathReview

[17] K Honda, W Kazez, G Matić, Tight contact structures on fibred hyperbolic 3-manifolds, J. Differential Geom. 64 (2003) 305-358 MathReview

[18] W Jaco, Lectures on three-manifold topology, CBMS Regional Conference Series in Mathematics 43, Amer. Math. Soc. Providence, RI (1980) MathReview

[19] Y Kanda, The classification of tight contact structures on the 3-torus, Comm. in Anal. and Geom. 5 (1997) 413-438 MathReview

[20] Y Kanda, On the Thurson-Bennequin invariant of Legendrian knots and non exactness of Bennequin's inequality, Invent. Math. 133 (1998) 227-242 MathReview

[21] S Makar-Limanov, Tight contact structures on solid tori, Trans. Amer. Math. Soc. 350 (1998) 1045-1078 MathReview

[22] J Martinet, Formes de contact sur les variétés de dimension 3, from: "Proceedings of Liverpool Singularities Symposium, II (1969/1970)", Lect. Notes in Math. 209, Springer (1971) 142-163 MathReview

Department of Mathematics, Northeastern Illinois University

5500 North St Louis Avenue, Chicago, IL 60625-4699, USA

Email: T-Cofer@neiu.edu

URL: http://www.neiu.edu/ tcofer/

Received: 9 November 2003 Revised: 20 May 2004 